\title{
Effect of Salinity Variation on the Quantity of Antioxidant Enzymes in Some Rice Cultivars of North Kerala, India
}

\author{
EA Joseph $^{1}$, KV Mohanan ${ }^{1,2, *}$, VV Radhakrishnan ${ }^{2}$ \\ ${ }^{1}$ Interuniversity Centre for Plant Biotechnology, Department of Botany, University of Calicut, India \\ ${ }^{2}$ Genetics and Plant Breeding Division, Department of Botany, University of Calicut, India
}

Copyright (C) 2015 Horizon Research Publishing All rights reserved.

\begin{abstract}
Rice (Oryza sativa L.) is one of the most popular and important cereal crops, providing the staple food for more than half of the world's population, especially those living in developing countries. Salinity is one of the prominent abiotic constraints for crop plants worldwide. It affects their growth, development, productivity and ultimately the yield. It also triggers a series of morphological, physiological, biochemical and molecular changes in plants. Various abiotic stresses lead to the overproduction of reactive oxygen species (ROS) in plants which are highly reactive and toxic and may cause severe damage to proteins, lipids, carbohydrates and DNA which ultimately results in oxidative stress. Plants possess very efficient enzymatic and non-enzymatic antioxidant defense systems which work together to control uncontrolled oxidation and protect plant cells from oxidative damage by the scavenging of ROS. The major antioxidant enzymes include catalase, peroxidase, super oxide dismutase, glutathione reductase, glutathione peroxidase, ascorbate peroxidase, etc. Production of antiaoxidant enzymes by some native rice genotypes collected both from saline and non-saline rice tracts of Kerala State of Inda was studied presently in relation to relative induction of salt stress. All the genotypes showed significant upregulation in the production of antioxidant enzymes in response to increase in salt stress applied.
\end{abstract}

Keywords Antioxidant Enzymes, Rice Cultivars, Oryza sativa, Reactive Oxygen Species, Salinity Stress

\section{Introduction}

Plants continually encounter an array of abiotic stresses like drought, salinity, heat and cold shock and heavy metals and these stresses constantly result in decrease in growth, development and productivity $[1,2,3,4]$. Among these stresses, salinity in soil or water is one of the major factors that limit crop production $[5,6,7,8,9]$. Stress induced cellular and metabolic changes result in enhanced accumulation of toxic compounds in cells including reactive oxygen species (ROS) [10]. The detrimental effects of salt on plants are consequences of both water deficit and interference in biochemical processes. Organisms that thrive in hyper saline environments possess various mechanisms including osmotic adjustment, exclusion of sodium ion and sequestration of sodium ion in the vacuole to avoid these effects. The capability to accumulate organic compatible solutes is very essential for osmoregulation in plants under high salinity and other stresses [11,12,13]. Salinity alters a wide array of metabolic processes in growing plants and induces changes in the contents and activities of many enzymes $[14,15,16,17]$.

Rice (Oryza sativa L.) is one of the most important cereal crops cultivated throughout the world and is regarded as the primary staple food for over two billion people worldwide [18]. It is a species capable of growing in swamps and freshwater marshes [19]. Rice plants are relatively susceptible to soil salinity and accumulation of salts in soil or water results in abiotic stress, a primary factor depressing crop production [20,21]. ROS are potentially harmful to cell, since they can raise the level of oxidative damage through loss of cellular structure and altering cellular functions. They are produced in many aerobic cellular metabolic processes. Cells possess various antioxidants and anti-oxidative enzymes to interrupt the cascades of uncontrolled oxidation both in the cell and in sub cellular organelles [22,23,24]. ROS are toxic to cell, whereas lower levels are necessary for the regulation of several key physiological mechanisms including cell differentiation [25], apoptosis [26], cell proliferation [27] and regulation of various redox-sensitive signal transduction pathways [28]. Plants have evolved both enzymatic and non-enzymatic mechanisms for ROS scavenging $[29,30]$. They use a diverse array of antioxidant enzymes like superoxide dismutase (SOD) [29], catalase (CAT) [31], peroxidases (POX) [32], ascorbate peroxidase (APX), glutathione reductase (GR), monodehydro ascorbate reductase (MDAR), dehydroascorbate reductase (DHAR) [29] and glutathione peroxidase (GPX) [33] as well as low molecular mass antioxidants such as ascorbate (ASC), reduced glutathione (GSH), oxidized glutathione (GSSG), phenolics and vitamins to scavenge different types of ROS [34,35,36,37].

Among all the antioxidative enzymes, superoxide dismutase and ascorbate peroxidase play crucial roles in ROS detoxification in cells. APX reduces $\mathrm{H}_{2} \mathrm{O}_{2}$ to water 
using ascorbic acid as a specific electron donor [38,39,40]. Chloroplasts are highly sensitive to damage by ROS that are frequently generated by the reaction of chloroplast $\mathrm{O}_{2}$ and electrons that escape from the photosynthetic electron transfer system [39]. To cope with this ROS-induced oxidative damage, plants employ the choloroplastic ROS scavenging enzymes copper-zinc SOD (Cu-Zn SOD) and APX. The role of these antioxidative enzymes at the onset of oxidative stress in chloroplasts has been extensively characterized [40].

The present experiment was carried out so as to study the variation in the quantity of antioxidant enzymes induced by salinity stress in the case of seven native rice cultivars of Kerala State of India, five collected from a traditional saline rice tract and two from a traditional non saline rice tract.

\section{Materials and Methods}

\subsection{Plant Materials and Seed Germination}

The experiment was conducted in the experimental rainout poly house of Department of Botany, University of Calicut, Kerala, India located at $11^{\circ} 35^{\prime} \mathrm{N}$ latitude and $75^{\circ} 48^{\prime} \mathrm{E}$ longitude in the first crop season of 2013. Seven native cultivars of rice including five cultivars namely Orthadian, Orkazhama, Kuthiru, Kuttusan and Chovvarian collected from one of the saline rice habitats of Kerala and two native rice cultivars namely Kunhutty and Veliyan collected from one of the non-saline rice habitats of Kerala were used for the study. Healthy mature caryopses from a single plant per cultivar were taken and washed in running tap water to remove unfilled grains and dust particles. The seeds were soaked in distilled water and allowed to germinate in $10 \mathrm{~cm}$ diameter Petri dishes covered with a lid under room temperature. The water was changed every day. The seeds started to germinate from the third day. On the 10th day, required number of germinated seedlings was transferred to coloured plastic pots of $25 \mathrm{~cm}$ diameter filled with paddy soil mixed with enriched compost in 3:1 ratio. Two seedlings were initially planted per pot and after the establishment of the seedlings the smallest among the two were removed. The plants were maintained in the experimental poly house under wetland conditions, always maintaining $3 \mathrm{~cm}$ of water above the soil level. The soil was fertilized with $1 \mathrm{~g}$ N:P:K $=18: 18: 18$ per pot at fortnightly intervals starting from the $30^{\text {th }}$ day. Weeding was done manually whenever required. Plants were grown in plastic pots of $25 \mathrm{~cm}$ diameter in Randomized Block Design with three replications.

\subsection{Experimental Treatments and Observations}

The experimental treatments were started from the $45^{\text {th }}$ day onwards, starting from $10 \mathrm{mM}\left(0.91 \mathrm{dSm}^{-1}\right)$ aqueous solution of sodium chloride and progressing up to $200 \mathrm{mM}$ $\left(18.26 \mathrm{dSm}^{-1}\right)$ as detailed in Table 1 , mimicking the progressive change in salinity in the traditional saline rice tracts of the study area.
Table 1. Salinity treatment details

\begin{tabular}{|c|c|}
\hline Treatment & Description \\
\hline $\mathrm{T} 1$ & Control \\
\hline $\mathrm{T} 2$ & $10 \mathrm{mM}\left(0.91 \mathrm{dSm}^{-1}\right)$ on $45^{\text {th }}$ day \\
\hline $\mathrm{T} 3$ & $\begin{array}{c}10 \mathrm{mM}\left(0.91 \mathrm{dSm}^{-1}\right) \text { on } 45^{\text {th }} \text { day } \& \\
30 \mathrm{mM}\left(2.74 \mathrm{dSm}^{-1}\right) \text { on } 53^{\text {rd }} \text { day }\end{array}$ \\
\hline $\mathrm{T} 4$ & $\begin{array}{c}10 \mathrm{mM}\left(0.91 \mathrm{dSm}^{-1}\right) \text { on } 45^{\text {th }} \text { day, } \\
30 \mathrm{mM}\left(2.74 \mathrm{dSm}^{-1}\right) \text { on } 53^{\text {rd }} \text { day } \& \\
50 \mathrm{mM}\left(4.57 \mathrm{dSm}^{-1}\right) \text { on } 61^{\text {st }} \text { day }\end{array}$ \\
\hline T5 & 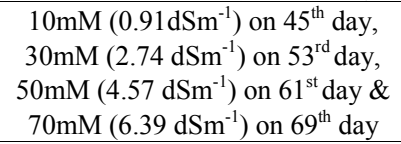 \\
\hline T6 & $\begin{array}{c}10 \mathrm{mM}\left(0.91 \mathrm{dSm}^{-1}\right) \text { on } 45^{\text {th }} \text { day, } \\
30 \mathrm{mM}^{-1}\left(2.74 \mathrm{dSm}^{-1}\right) \text { on } 53^{\text {rd }} \text { day, } \\
50 \mathrm{mM}\left(4.57 \mathrm{dSm}^{-1}\right) \text { on } 61^{\text {st }} \text { day, } \\
70 \mathrm{mM}\left(6.39 \mathrm{dSm}^{-1}\right) \text { on } 69^{\text {th }} \text { day } \& \\
100 \mathrm{mM}^{\text {th }}\left(9.13 \mathrm{dSm}^{-1}\right) \text { on } 77^{\text {th }} \text { day }\end{array}$ \\
\hline $\mathrm{T} 7$ & 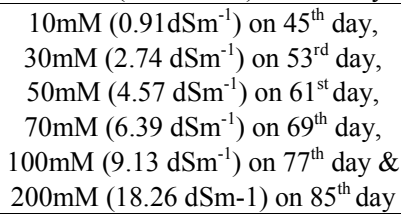 \\
\hline
\end{tabular}

\subsubsection{Enzyme extraction}

On the $90^{\text {th }}$ day the leaves of the treated and control plants were used for the enzyme assays. Enzyme extraction was carried out according to Larkindale and Huang, 2004 [41]. Harvested fresh rice leaves $(0.25 \mathrm{~g})$ were crushed into fine powder in a pre-chilled mortar and pestle with liquid nitrogen. Further, this powder was homogenized in cold phosphate buffer $(1.5 \mathrm{ml}$ of $100 \mathrm{mM}, \mathrm{pH} 7.0)$ containing polyvinyl pyrrolidone (1\% PVP, (w/v) Himedia, India) and EDTA (1mM, Himedia, India) and centrifuged at $10,000 \times \mathrm{g}$ for 15 minutes at $4^{\circ} \mathrm{C}$ (Sigma, Germany). Supernatant was stored on ice for all enzymatic assays. In the case of APX assay, the extraction buffer was supplemented with L-ascorbate (2mM, Himedia, India).

\subsubsection{Assay of catalase}

Catalase (CAT) [EC 1.11.1.6] estimation was done according to Aebi, 1983 [42]. $3 \mathrm{ml}$ reaction mixture consisting of diluted enzyme extract $(100 \mu \mathrm{l})$ and potassium phosphate buffer $(2.8 \mathrm{ml}$ of $50 \mathrm{mM}, \mathrm{pH} 7.0)$ was mixed. Reaction was initiated by adding $\mathrm{H}_{2} \mathrm{O}_{2}(100 \mu \mathrm{l}$ of $100 \mathrm{mM}$, Merck). Change in absorbance was recorded spectrophotometrically at $240 \mathrm{~nm}$ at an interval of 15 seconds for 2 minutes. Activity of catalase was estimated by monitoring the decrease in absorbance due to $\mathrm{H}_{2} \mathrm{O}_{2}$ reduction at $240 \mathrm{~nm}$. One unit of enzyme activity is defined as the amount of enzyme that catalyzes the decomposition of $1 \mu \mathrm{M}$ of $\mathrm{H}_{2} \mathrm{O}_{2}$ per min at $\mathrm{pH} 7.0$ under standard conditions $\left(25 \pm 2^{\circ} \mathrm{C}\right)$. The enzyme activity was expressed as units $\mathrm{min}^{-1}$ mg protein ${ }^{-1}$.

\subsubsection{Assay of peroxidase}

Peroxidase (POX) activity [EC 1.11.1.7] was assayed and estimated as per the procedure of Rao et al., 1996 [43] with slight modifications. POX activity was measured using the oxidation of guaiacol used as substrate. For assay, the 
reaction mixture consisted of $1.7 \mathrm{ml}$ of $100 \mathrm{mM}$ potassium phosphate buffer ( $\mathrm{pH} 7.0)$ containing EDTA $(1 \mathrm{mM}), 0.1 \mathrm{ml}$ of $20 \mathrm{mM}$ guaiacol (Himedia), $100 \mu \mathrm{l}$ of $10 \mathrm{mM} \mathrm{H}_{2} \mathrm{O}_{2}$ and $100 \mu 1$ of the enzyme extract. Reaction was initiated by adding $\mathrm{H}_{2} \mathrm{O}_{2}$. The increase in absorbance was measured spectrophotometrically at $470 \mathrm{~nm}$ for 2 minutes with 15 second intervals at $30^{\circ} \mathrm{C}$. A set of samples containing the reaction mixture without guaiacol was taken as the control. One unit of POX activity is the amount of enzyme required to oxidize $1 \mu \mathrm{M}$ of guaiacol by $\mathrm{H}_{2} \mathrm{O}_{2}$ at that condition. Enzyme activity was expressed as units $\mathrm{min}^{-1} \mathrm{mg}$ protein ${ }^{-1}$.

\subsubsection{Assay of glutathione reductase}

Glutathione reductase (GR) [EC 1.6.4.2] activity was estimated by the method of Foyer and Halliwell (1986) [44] and Smith et al. (1988) [45] with a little modification. The activity of GR was determined by following the oxidation of NADPH. The reagents used were potassium phosphate buffer (100mM, pH 7.6) containing EDTA (1mM, SRL), NADPH (5mM, Sigma), 5, 5'-dithio-bis 2-nitrobenzoic acid (6mM DTNB, SRL) and oxidized glutathione $(0.2 \mathrm{mM}$ GSSG, Himedia). The $3 \mathrm{ml}$ reaction mixture consisted of potassium phosphate buffer $(2.65 \mathrm{ml})$, diluted enzyme extract $(150 \mu \mathrm{l})$ and DTNB $(100 \mu \mathrm{l})$, and reaction was started by adding GSSG $(50 \mu \mathrm{l})$. Reaction was initiated by the addition of $50 \mu \mathrm{l}$ of NADPH. Change in absorbance at $412 \mathrm{~nm}$ was followed at 15 second intervals up to 2 minutes. A change in absorbance by 0.01 corresponds to 1 unit and enzyme activity was expressed as units $\min ^{-1} \mathrm{mg}$ protein ${ }^{-1}$. One unit of GR was defined as $1 \mathrm{mmol} \mathrm{ml}{ }^{-1}$ GSSG reduced per minute.

\subsubsection{Assay of glutathione peroxidase (GPX)}

Glutathione peroxidase (GPX) [EC 1.11.1.9] was assayed with hydrogen peroxide and t-butyl hydroperoxide as substrates following the modified procedure of Flohe and Gunzler (1984) [46] and Inoue et al. (1999) [47] with minor modifications. GPX activity was measured in a reaction mixture $(3.0 \mathrm{ml})$ composed of potassium phosphate buffer (1.5ml of $100 \mathrm{mM}, \mathrm{pH} 7.6)$ with EDTA (1mM, SRL), GSH ( $0.3 \mathrm{ml}$ of $10 \mathrm{mM}$, Himedia), NADPH $(0.3 \mathrm{ml}$ of $2 \mathrm{mM}$, SRL), GR (0.6units, SRL), and enzyme extract $(0.3 \mathrm{ml})$. The reaction mixture was pre-incubated at $37^{\circ} \mathrm{C}$ for 10 minutes, and the reaction was started by addition of pre warmed t-butyl hydroperoxide ( $0.3 \mathrm{ml}$, Merck). Change in absorbance at $340 \mathrm{~nm}$ was followed at 15 second intervals up to 2 minutes using spectrophotometer. A change of 0.1 absorbance has been taken as 1unit. Enzyme activity was expressed as units $\min ^{-1} \mathrm{mg}$ protein ${ }^{-1}$. One unit of activity was defined as the consumption of $1 \mathrm{mmol} \mathrm{NADPH}$ per minute.

\subsubsection{Assay of ascorbate peroxidase (APX)}

Ascorbate peroxidase (APX) [EC 1.11.1.11] activity was measured by the method of Nakano and Asada (1981) [48]. $3 \mathrm{ml}$ reaction mixture containing diluted enzyme extract $(0.1$ $\mathrm{ml}$ ) and potassium phosphate buffer $(1.5 \mathrm{ml}$ of $50 \mathrm{mM}, \mathrm{pH} 7.0)$ containing ascorbic acid $(0.5 \mathrm{mM}$, Himedia) was taken. The reaction was started by the addition of $\mathrm{H}_{2} \mathrm{O}_{2}(0.4 \mathrm{ml}$ of $1 \mathrm{mM}$,
Merck), and the decrease in absorbance was recorded at $290 \mathrm{~nm}$ at 15 seconds intervals for 2 minutes. A change in absorbance by 0.01 corresponded to 1 unit of enzyme activity Enzyme activity was expressed as units $\min ^{-1} \mathrm{mg}_{\text {protein }}{ }^{-1}$. One unit of APX was defined as $1 \mathrm{nmol} \mathrm{m} \mathrm{m}^{-1}$ ascorbate oxidized per minute.

\subsubsection{Assay of superoxide dismutase (SOD)}

SOD [EC 1.15.1.1] activity was estimated using xanthine-Xanthine oxidase system as suggested by Beyer and Fridovich (1987) [49] with modifications. Superoxide dismutase (SOD, EC 1.15.1.1) activity was assayed spectrophotometrically as the inhibition of photochemical reduction of nitro-blue tetrazolium (NBT) at $560 \mathrm{~nm}$. Reagents used for assay included potassium phosphate buffer (50mM, pH 7.8), catalase (Sigma), nitroblue tetrazolium (2.24mM, NBT, SRL), xanthine (2.36mM, SRL) and xanthine oxidase (Sigma). SOD activity was assayed based on its ability to compete with NBT for superoxide anions generated by the xanthine-xanthine oxidase system, which, in turn, results in the inhibition of reduction of NBT. Catalase (0.1 units) was added to the assay mixture to avoid the $\mathrm{H}_{2} \mathrm{O}_{2}$-mediated possible inactivation of $\mathrm{Cu} / \mathrm{Zn}-\mathrm{SOD}$. Diluted enzyme extract $(100 \mu \mathrm{l})$ was taken, and to it, potassium phosphate buffer $(800 \mu \mathrm{l})$, NBT $(50 \mu \mathrm{l}, \mathrm{SRL})$, catalase (0.1 units, Himedia), and xanthine oxidase (0.1 units, Sigma) were added. Reaction was initiated by adding $50 \mu$ of xanthine. Change in absorbance was read at $560 \mathrm{~nm}$ up to 2 minutes. A blank reaction was performed using all the components but sample extracts to get the maximum colour. Enzyme activity was calculated in units (i.e., amount of enzyme required to inhibit NBT reduction by $50 \%$ under specified conditions of the assay) and expressed as units $\min ^{-1} \mathrm{mg}$ protein ${ }^{-1}$.

\subsubsection{Estimation of total soluble protein}

Estimation of total soluble protein was done according to the method advocated by Lowry et al., (1951) [50] as detailed below:

Reagents used: A- 2\% Sodium carbonate in $0.1 \mathrm{~N}$ Sodium hydroxide; B- $0.5 \%$ Copper sulphate in $1 \%$ Potassium sodium tartarate, C- Alkaline Copper sulphate solution: Mixed $50 \mathrm{ml} \mathrm{A}$ and $1 \mathrm{ml}$ of $\mathrm{B}$ prior to use; D Folin-Ciocalteau reagent.

Procedure: Suitable aliquots were taken in duplicates from each preparation. Volume was made up to $1 \mathrm{ml}$ with double distilled water. Then $5 \mathrm{ml}$ of reagent $\mathrm{C}$ was added to each tube, mixed well and kept at room temperature for 10 minutes and $0.5 \mathrm{ml} 1 \mathrm{~N}$ Folin-Ciocalteau reagent was added with immediate mixing. The tubes were kept for 30 minutes for colour development. Absorbance (Optical Density) was read at $700 \mathrm{~nm}$ using a UV-Visible spectrophotometer (Thermo Scientific, USA).

\subsection{Statistical Analysis}

The experiments were carried out in randomized block design (RBD) with three replications. Data were statistically analysed by ANOVA to find out the significance of variations and are presented as mean \pm standard error (SE) at $\mathrm{P} \geq 0.05$. 
Table 2. Activity and percentage of increase in activity in the case of catalase in different rice cultivars under salinity stress.

\begin{tabular}{|c|c|c|c|}
\hline Cultivar/ Treatment & Mean \pm SE & $\mathrm{CD} @ 5 \%$ & Percentage of increase over control \\
\hline \multicolumn{4}{|c|}{ Orthadian } \\
\hline Control & $3.27 \pm 0.03$ & \multirow{7}{*}{1.15} & - \\
\hline $10 \mathrm{mM}$ & $3.73 \pm 0.05$ & & 14.07 \\
\hline $30 \mathrm{mM}$ & $5.00 \pm 0.33^{*}$ & & 52.91 \\
\hline $50 \mathrm{mM}$ & $6.73 \pm 0.14 *$ & & 105.81 \\
\hline $70 \mathrm{mM}$ & $7.80 \pm 0.04 *$ & & 138.53 \\
\hline $100 \mathrm{mM}$ & $8.33 \pm 0.05^{*}$ & & 154.74 \\
\hline $200 \mathrm{mM}$ & $8.47 \pm 0.09 *$ & & 159.02 \\
\hline \multicolumn{4}{|c|}{ Chovvarian } \\
\hline Control & $3.00 \pm 0.04$ & \multirow{7}{*}{1.00} & - \\
\hline $10 \mathrm{mM}$ & $3.60 \pm 0.08$ & & 20.00 \\
\hline $30 \mathrm{mM}$ & $4.87 \pm 0.13^{*}$ & & 62.33 \\
\hline $50 \mathrm{mM}$ & $5.73 \pm 0.20^{*}$ & & 91.00 \\
\hline $70 \mathrm{mM}$ & $6.93 \pm 0.13 *$ & & 131.00 \\
\hline $100 \mathrm{mM}$ & $7.73 \pm 0.14^{*}$ & & 157.67 \\
\hline $200 \mathrm{mM}$ & $8.20 \pm 0.09 *$ & & 173.33 \\
\hline \multicolumn{4}{|c|}{ Kuttusan } \\
\hline Control & $3.07 \pm 0.07$ & \multirow{7}{*}{0.79} & - \\
\hline $10 \mathrm{mM}$ & $3.73 \pm 0.05$ & & 21.50 \\
\hline $30 \mathrm{mM}$ & $4.93 \pm 0.17 *$ & & 60.59 \\
\hline $50 \mathrm{mM}$ & $6.07 \pm 0.05^{*}$ & & 91.72 \\
\hline $70 \mathrm{mM}$ & $7.13 \pm 0.18^{*}$ & & 132.25 \\
\hline $100 \mathrm{mM}$ & $8.00 \pm 0.00 *$ & & 160.59 \\
\hline $200 \mathrm{mM}$ & $8.47 \pm 0.03 *$ & & 175.90 \\
\hline \multicolumn{4}{|c|}{ Kuthiru } \\
\hline Control & $3.27 \pm 0.10$ & \multirow{7}{*}{0.68} & - \\
\hline $10 \mathrm{mM}$ & $3.47 \pm 0.03$ & & 6.12 \\
\hline $30 \mathrm{mM}$ & $5.13 \pm 0.03 *$ & & 56.88 \\
\hline $50 \mathrm{mM}$ & $6.93 \pm 0.15^{*}$ & & 111.93 \\
\hline $70 \mathrm{mM}$ & $8.00 \pm 0.04 *$ & & 144.65 \\
\hline $100 \mathrm{mM}$ & $8.47 \pm 0.03 *$ & & 159.02 \\
\hline $200 \mathrm{mM}$ & $8.80 \pm 0.12 *$ & & 169.11 \\
\hline \multicolumn{4}{|c|}{ Orkazhama } \\
\hline Control & $2.80 \pm 0.04$ & \multirow{7}{*}{0.95} & - \\
\hline $10 \mathrm{mM}$ & $3.60 \pm 0.04$ & & 28.57 \\
\hline $30 \mathrm{mM}$ & $4.73 \pm 0.28^{*}$ & & 68.93 \\
\hline $50 \mathrm{mM}$ & $6.33 \pm 0.09^{*}$ & & 126.07 \\
\hline $70 \mathrm{mM}$ & $7.67 \pm 0.05^{*}$ & & 173.93 \\
\hline $100 \mathrm{mM}$ & $8.07 \pm 0.03 *$ & & 188.21 \\
\hline $200 \mathrm{mM}$ & $8.33 \pm 0.07 *$ & & 197.50 \\
\hline \multicolumn{4}{|c|}{ Kunhutty } \\
\hline Control & $2.20 \pm 0.04$ & \multirow{7}{*}{0.76} & - \\
\hline $10 \mathrm{mM}$ & $2.47 \pm 0.03$ & & 12.27 \\
\hline $30 \mathrm{mM}$ & $3.60 \pm 0.16^{*}$ & & 63.64 \\
\hline $50 \mathrm{mM}$ & $3.87 \pm 0.09^{*}$ & & 75.91 \\
\hline $70 \mathrm{mM}$ & $4.93 \pm 0.03 *$ & & 124.09 \\
\hline $100 \mathrm{mM}$ & $6.27 \pm 0.15^{*}$ & & 185.00 \\
\hline $200 \mathrm{mM}$ & $7.20 \pm 0.08^{*}$ & & 227.27 \\
\hline \multicolumn{4}{|c|}{ Veliyan } \\
\hline Control & $2.33 \pm 0.03$ & \multirow{7}{*}{0.83} & - \\
\hline $10 \mathrm{mM}$ & $2.93 \pm 0.10$ & & 25.75 \\
\hline $30 \mathrm{mM}$ & $3.80 \pm 0.00^{*}$ & & 63.09 \\
\hline $50 \mathrm{mM}$ & $4.93 \pm 0.11 *$ & & 111.59 \\
\hline $70 \mathrm{mM}$ & $5.67 \pm 0.22 *$ & & 143.35 \\
\hline $100 \mathrm{mM}$ & $7.27 \pm 0.03 *$ & & 212.02 \\
\hline $200 \mathrm{mM}$ & $7.73 \pm 0.03 *$ & & 231.76 \\
\hline
\end{tabular}

*Significant variation from the control at $5 \%$ level 


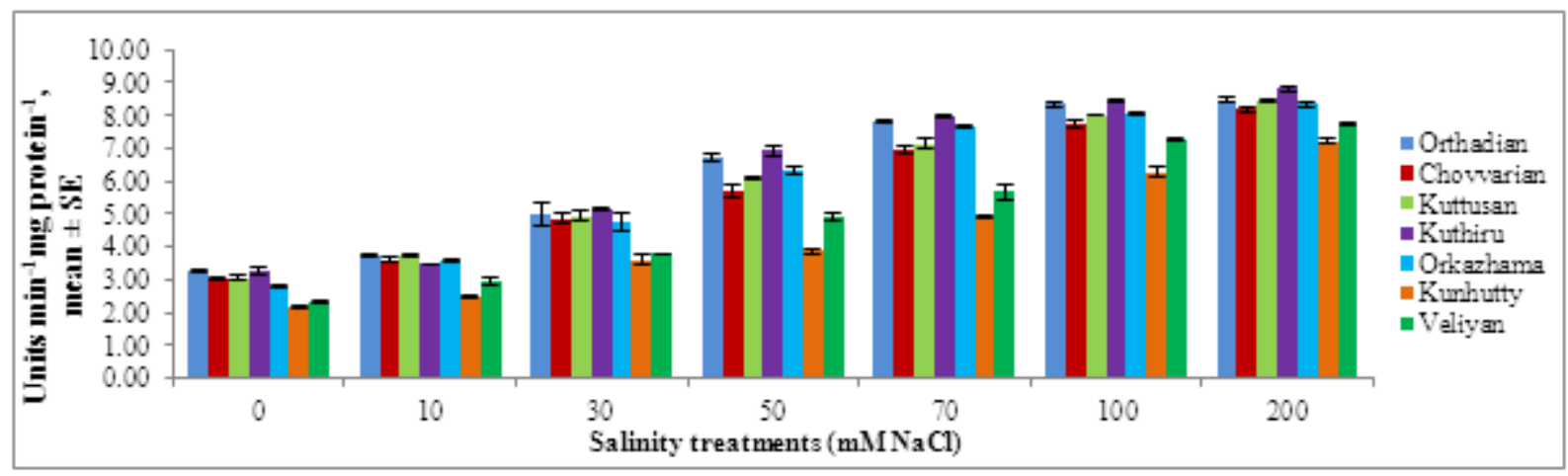

Figure 1. Activity of catalase in different rice genotypes under different salt concentrations

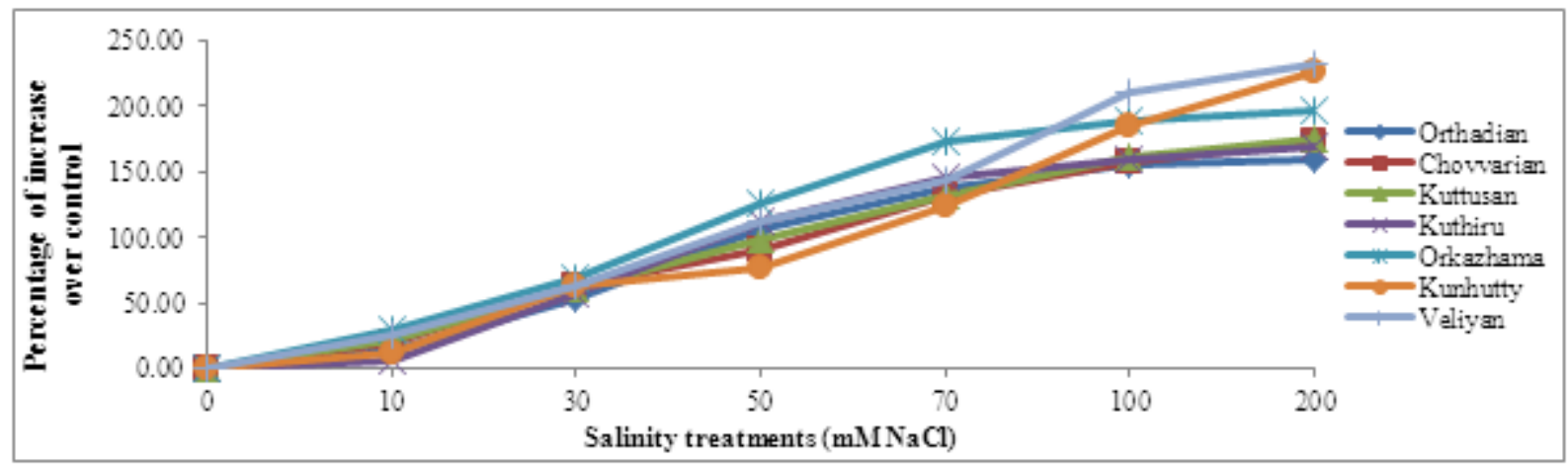

Figure 2. Increase in catalase activity in different rice genotypes under different salt concentrations

\section{Results}

The activity and the percentage of increase in enzyme activity in the case of the different enzymes studied showed that there was significant increase in the activity of all the enzymes except glutathione peroxidase under gradual increase in the level of salinity.

\subsection{Activity of Catalase}

Catalase activity increased significantly starting from $30 \mathrm{mM}$ salinity treatment in all the rice genotypes studied. The percentage of increase in the activity also increased in relation to raise in salt concentration (Table 2, Figs. $1 \& 2$ ). The highest activity and the highest percentage of increase in activity were noticed in $200 \mathrm{mM}$ salt concentration in all the cultivars. The highest level of catalase activity was shown by the cultivar Kuthiru followed by Orthadian, Kuttusan, Orkazhama,Chovvarian, Veliyan and Kunhutty in that order. The first five of the above were collected from a saline rice tract and others from a non-saline rice tract. This shows that the rice cultivars collected from the saline rice tract showed higher catalase activity under salt stress when compared to the cultivars collected from the non-saline rice tract. The highest percentage of increase in catalase activity was observed in Veliyan (231.76\%), a cultivar collected from the non-saline rice tract.

\subsection{Activity of Peroxidase}

The highest peroxidase enzyme activity was shown by the rice cultivar Kuthiru collected from the saline rice tract followed by Orthadian, Chovvarian, Veliyan, Kuttusan, Orkazhama and Kunhutty (Table 3; Figs. 3 \& 4). Peroxidase activity significantly increased from $30 \mathrm{mM}$ salt treatment in Orthadian, Chovvarian and Kuttusan and from 50mM in all the other genotypes. The percentage of increase in peroxidase activity also increased with increase in salinity levels and the percentage of increase was the highest in Chovvarian $(77.57 \%)$, a cultivar collected from the saline rice tract followed by Veliyan, Kuthiru, Orthadian, Kuttusan, Orkazhama and Kunhutty. 
Table 3. Activity and percentage of increase in activity in the case of peroxidase in different rice cultivars under salinity stress.

\begin{tabular}{|c|c|c|c|}
\hline Cultivar/ Treatment & Mean \pm SE & $\mathrm{CD} @ 5 \%$ & Percentage of increase over control \\
\hline \multicolumn{4}{|c|}{ Orthadian } \\
\hline Control & $41.80 \pm 0.27$ & \multirow{7}{*}{2.13} & - \\
\hline $10 \mathrm{mM}$ & $42.33 \pm 0.14$ & & 1.27 \\
\hline $30 \mathrm{mM}$ & $44.87 \pm 0.03 *$ & & 7.34 \\
\hline $50 \mathrm{mM}$ & $49.20 \pm 0.31 *$ & & 17.70 \\
\hline $70 \mathrm{mM}$ & $54.93 \pm 0.13^{*}$ & & 31.41 \\
\hline $100 \mathrm{mM}$ & $62.53 \pm 0.39^{*}$ & & 49.59 \\
\hline $200 \mathrm{mM}$ & $72.87 \pm 0.36^{*}$ & & 74.33 \\
\hline \multicolumn{4}{|c|}{ Chovvarian } \\
\hline Control & $39.53 \pm 0.29$ & \multirow{7}{*}{3.08} & - \\
\hline $10 \mathrm{mM}$ & $40.67 \pm 0.29$ & & 2.88 \\
\hline $30 \mathrm{mM}$ & $43.67 \pm 0.07 *$ & & 10.47 \\
\hline $50 \mathrm{mM}$ & $49.33 \pm 0.40^{*}$ & & 24.79 \\
\hline $70 \mathrm{mM}$ & $55.67 \pm 0.62 *$ & & 40.83 \\
\hline $100 \mathrm{mM}$ & $61.27 \pm 0.14 *$ & & 55.00 \\
\hline $200 \mathrm{mM}$ & $70.20 \pm 0.55^{*}$ & & 77.57 \\
\hline \multicolumn{4}{|c|}{ Kuttusan } \\
\hline Control & $39.80 \pm 0.17$ & \multirow{7}{*}{2.95} & - \\
\hline $10 \mathrm{mM}$ & $40.47 \pm 0.05$ & & 1.68 \\
\hline $30 \mathrm{mM}$ & $45.93 \pm 0.64 *$ & & 15.40 \\
\hline $50 \mathrm{mM}$ & $49.53 \pm 0.29 *$ & & 24.45 \\
\hline $70 \mathrm{mM}$ & $54.07 \pm 0.41^{*}$ & & 35.85 \\
\hline $100 \mathrm{mM}$ & $58.60 \pm 0.50 *$ & & 47.24 \\
\hline $200 \mathrm{mM}$ & $64.80 \pm 0.12 *$ & & 62.81 \\
\hline \multicolumn{4}{|c|}{ Kuthiru } \\
\hline Control & $43.20 \pm 0.20$ & \multirow{7}{*}{4.77} & - \\
\hline $10 \mathrm{mM}$ & $44.40 \pm 0.65$ & & 2.78 \\
\hline $30 \mathrm{mM}$ & $45.07 \pm 1.07$ & & 4.33 \\
\hline $50 \mathrm{mM}$ & $50.67 \pm 0.49^{*}$ & & 17.29 \\
\hline $70 \mathrm{mM}$ & $57.80 \pm 0.48^{*}$ & & 33.80 \\
\hline $100 \mathrm{mM}$ & $65.40 \pm 1.32 *$ & & 51.39 \\
\hline $200 \mathrm{mM}$ & $75.60 \pm 0.09 *$ & & 75.00 \\
\hline \multicolumn{4}{|c|}{ Orkazhama } \\
\hline Control & $39.47 \pm 0.18$ & \multirow{7}{*}{5.95} & - \\
\hline $10 \mathrm{mM}$ & $40.87 \pm 0.18$ & & 3.55 \\
\hline $30 \mathrm{mM}$ & $44.47 \pm 1.12$ & & 12.67 \\
\hline $50 \mathrm{mM}$ & $49.80 \pm 0.12 *$ & & 26.17 \\
\hline $70 \mathrm{mM}$ & $53.47 \pm 0.39 *$ & & 35.47 \\
\hline $100 \mathrm{mM}$ & $57.93 \pm 0.93 *$ & & 46.77 \\
\hline $200 \mathrm{mM}$ & $63.47 \pm 0.35^{*}$ & & 60.81 \\
\hline \multicolumn{4}{|c|}{ Kunhutty } \\
\hline Control & $34.73 \pm 0.13$ & \multirow{7}{*}{2.18} & - \\
\hline $10 \mathrm{mM}$ & $34.87 \pm 0.26$ & & 0.40 \\
\hline $30 \mathrm{mM}$ & $35.20 \pm 0.16$ & & 1.35 \\
\hline $50 \mathrm{mM}$ & $37.13 \pm 0.03 *$ & & 6.91 \\
\hline $70 \mathrm{mM}$ & $41.80 \pm 0.50^{*}$ & & 20.36 \\
\hline $100 \mathrm{mM}$ & $46.53 \pm 0.07 *$ & & 33.98 \\
\hline $200 \mathrm{mM}$ & $51.87 \pm 0.39^{*}$ & & 49.35 \\
\hline \multicolumn{4}{|c|}{ Veliyan } \\
\hline Control & $36.80 \pm 0.20$ & \multirow{7}{*}{4.01} & - \\
\hline $10 \mathrm{mM}$ & $37.00 \pm 0.43$ & & 0.54 \\
\hline $30 \mathrm{mM}$ & $39.20 \pm 0.31$ & & 6.52 \\
\hline $50 \mathrm{mM}$ & $43.27 \pm 0.41 *$ & & 17.58 \\
\hline $70 \mathrm{mM}$ & $50.87 \pm 1.08^{*}$ & & 38.23 \\
\hline $100 \mathrm{mM}$ & $56.87 \pm 0.29 *$ & & 54.54 \\
\hline $200 \mathrm{mM}$ & $65.00 \pm 0.08^{*}$ & & 76.63 \\
\hline
\end{tabular}

*Significant variation from the control at $5 \%$ level 


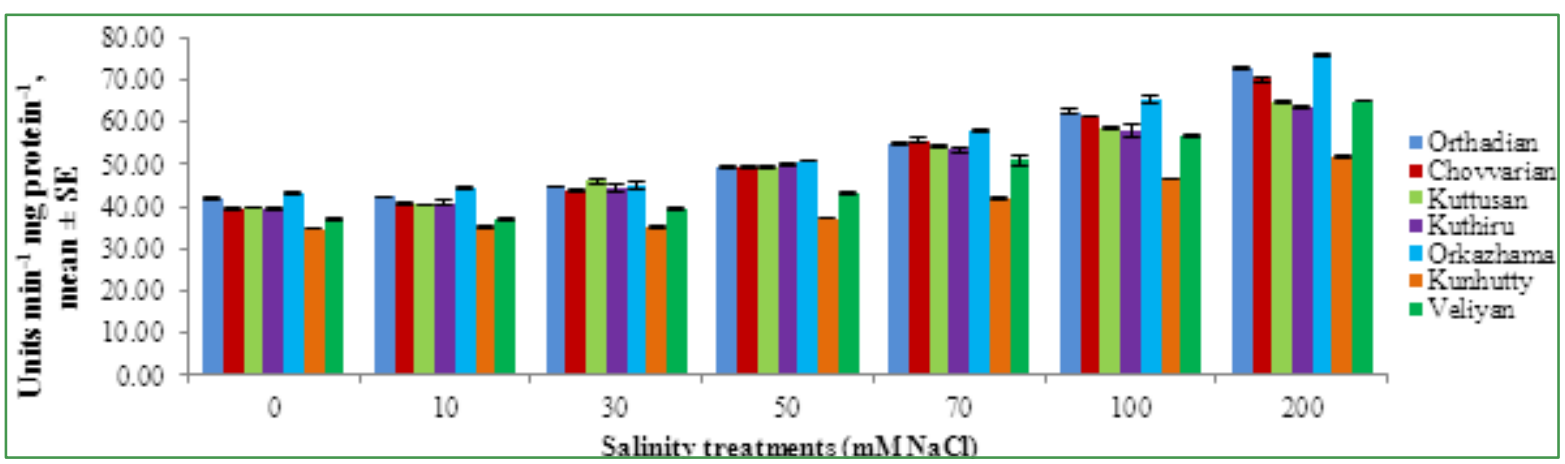

Figure 3. Activity of peroxidase in different rice genotypes under different salt concentrations

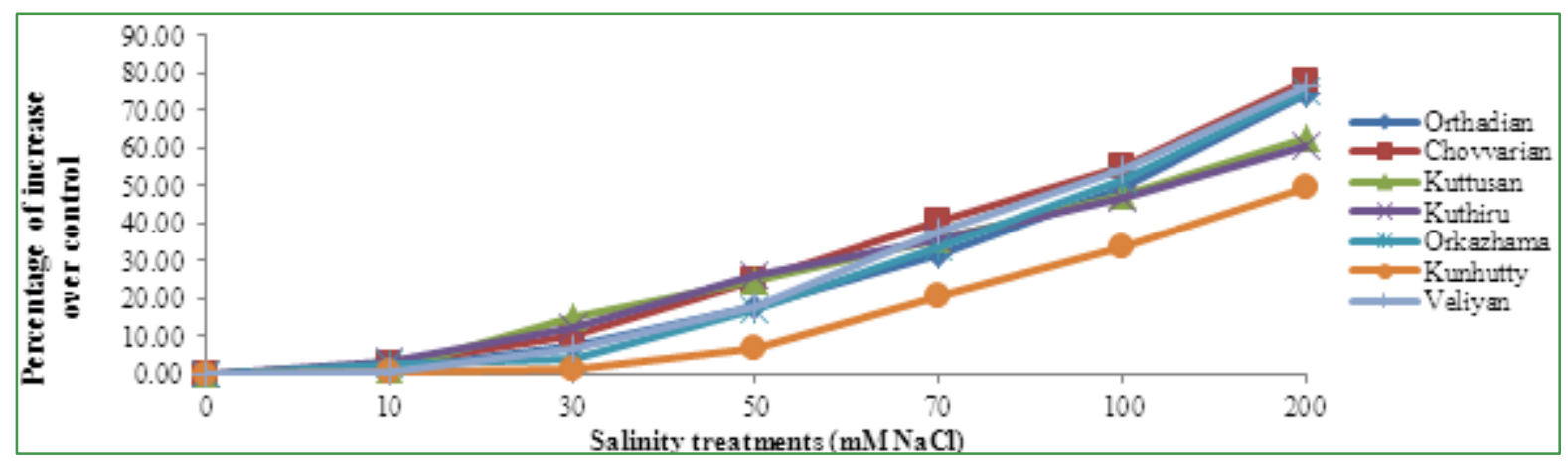

Figure 4. Increase in peroxidase activity in different rice genotypes under different salt concentrations

\subsection{Activity of Glutathione Reductase}

All the rice genotypes under study showed significant increase in glutathione reductase activity due to increase in salinity concentration in the medium (Table 4; Figs. 5 \& 6). Kuthiru showed the highest activity of glutathione peroxidase followed by Orthadian. Both of these are cultivars from the saline rice tract. The highest percentage of increase in the activity of glutathione reductase was also shown by the same genotypes in the above order (56.29\% and 55.95\%). Kunhutty, a cultivar from the non-saline rice tract showed significant increase in glutathione reductase activity from $30 \mathrm{mM}$ salt concentration onwards. Ortthadian, Kuthiru and Veliyan showed significant increase in activity of this enzyme from $50 \mathrm{mM}$ salt concentration and Chovvarian, Kuttusan, and Orkazhama showed variation from $70 \mathrm{mM}$ of salinity onwards.

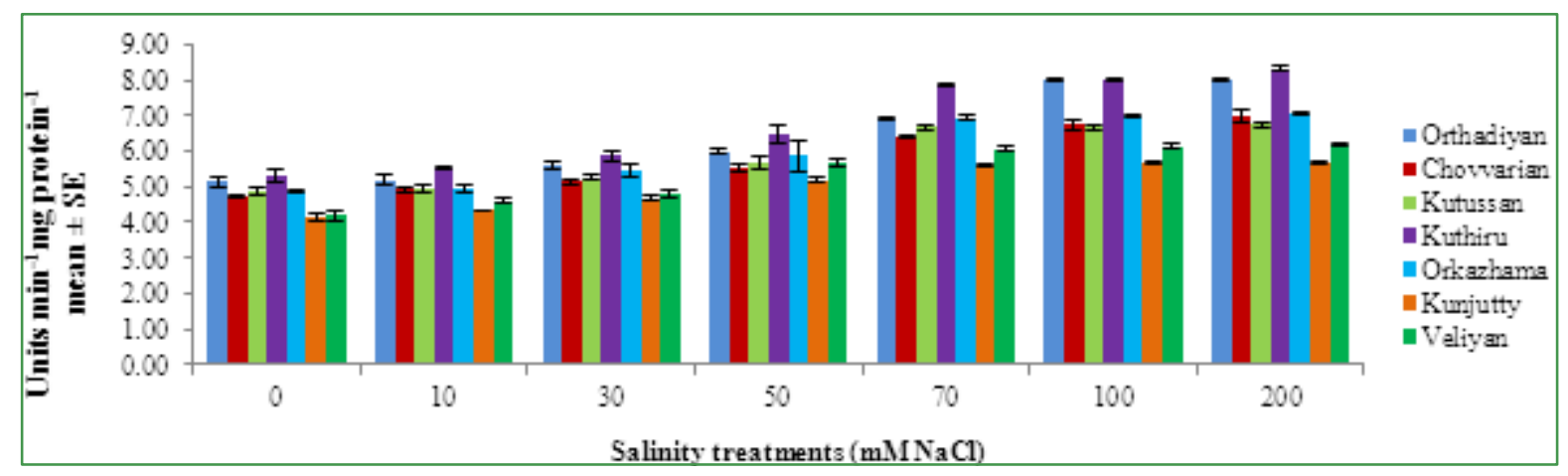

Figure 5. Activity of glutathione reductase in different rice genotypes under different salt concentrations

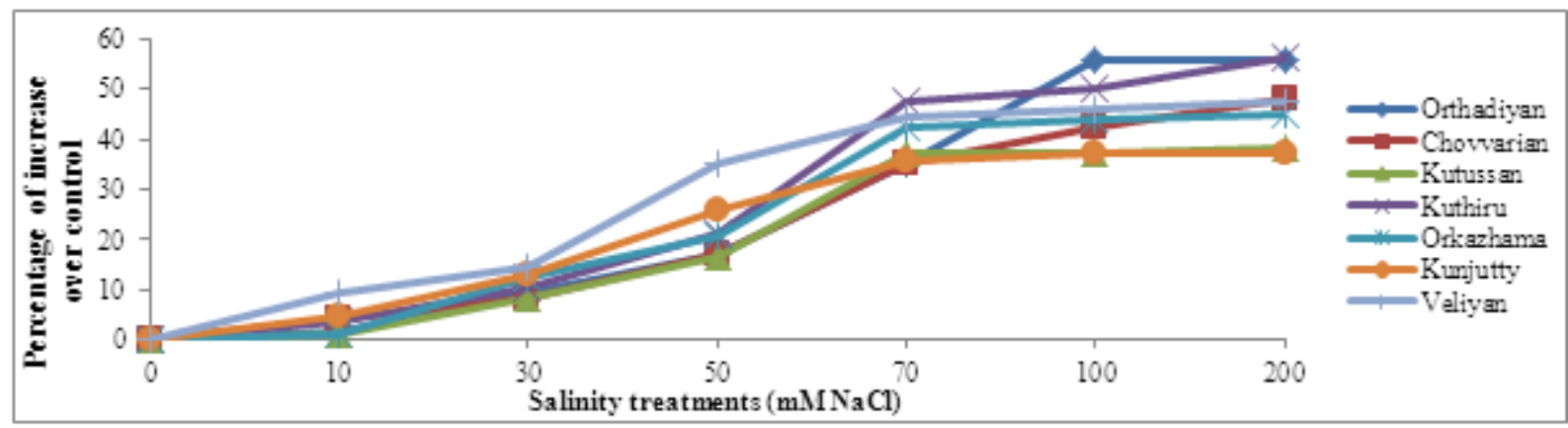

Figure 6. Increase in glutathione reductase activity in different rice genotypes under different salt concentrations 
Table 4. Activity and percentage of increase in activity in the case of glutathione reductase in different rice cultivars under salinity stress.

\begin{tabular}{|c|c|c|c|}
\hline Cultivar/ Treatment & Mean \pm SE & $\mathrm{CD} @ 5 \%$ & Percentage of increase over control \\
\hline \multicolumn{4}{|c|}{ Orthadian } \\
\hline Control & $5.13 \pm 0.18$ & \multirow{7}{*}{0.83} & - \\
\hline $10 \mathrm{mM}$ & $5.20 \pm 0.16$ & & 1.36 \\
\hline $30 \mathrm{mM}$ & $5.60 \pm 0.09$ & & 9.16 \\
\hline $50 \mathrm{mM}$ & $6.00 \pm 0.08^{*}$ & & 16.96 \\
\hline $70 \mathrm{mM}$ & $6.93 \pm 0.05^{*}$ & & 35.09 \\
\hline $100 \mathrm{mM}$ & $8.00 \pm 0.04 *$ & & 55.95 \\
\hline $200 \mathrm{mM}$ & $8.00 \pm 0.04 *$ & & 55.95 \\
\hline \multicolumn{4}{|c|}{ Chovvarian } \\
\hline Control & $4.73 \pm 0.03$ & \multirow{7}{*}{0.90} & - \\
\hline $10 \mathrm{mM}$ & $4.93 \pm 0.07$ & & 4.22 \\
\hline $30 \mathrm{mM}$ & $5.13 \pm 0.07$ & & 8.46 \\
\hline $50 \mathrm{mM}$ & $5.53 \pm 0.10$ & & 16.91 \\
\hline $70 \mathrm{mM}$ & $6.40 \pm 0.04 *$ & & 35.31 \\
\hline $100 \mathrm{mM}$ & $6.73 \pm 0.17 *$ & & 42.28 \\
\hline $200 \mathrm{mM}$ & $7.00 \pm 0.20^{*}$ & & 47.99 \\
\hline \multicolumn{4}{|c|}{ Kuttusan } \\
\hline Control & $4.87 \pm 0.09$ & \multirow{7}{*}{0.83} & - \\
\hline $10 \mathrm{mM}$ & $4.93 \pm 0.13$ & & 1.23 \\
\hline $30 \mathrm{mM}$ & $5.27 \pm 0.07$ & & 8.21 \\
\hline $50 \mathrm{mM}$ & $5.67 \pm 0.17$ & & 16.43 \\
\hline $70 \mathrm{mM}$ & $6.67 \pm 0.07^{*}$ & & 36.96 \\
\hline $100 \mathrm{mM}$ & $6.67 \pm 0.07 *$ & & 36.96 \\
\hline $200 \mathrm{mM}$ & $6.73 \pm 0.09 *$ & & 38.19 \\
\hline \multicolumn{4}{|c|}{ Kuthiru } \\
\hline Control & $5.33 \pm 0.18$ & \multirow{7}{*}{1.05} & - \\
\hline $10 \mathrm{mM}$ & $5.53 \pm 0.05$ & & 3.75 \\
\hline $30 \mathrm{mM}$ & $5.87 \pm 0.13$ & & 10.13 \\
\hline $50 \mathrm{mM}$ & $6.47 \pm 0.24 *$ & & 21.39 \\
\hline $70 \mathrm{mM}$ & $7.87 \pm 0.03^{*}$ & & 47.65 \\
\hline $100 \mathrm{mM}$ & $8.00 \pm 0.04 *$ & & 50.09 \\
\hline $200 \mathrm{mM}$ & $8.33 \pm 0.09 *$ & & 56.29 \\
\hline \multicolumn{4}{|c|}{ Orkazhama } \\
\hline Control & $4.87 \pm 0.03$ & \multirow{7}{*}{1.50} & - \\
\hline $10 \mathrm{mM}$ & $4.93 \pm 0.09$ & & 1.23 \\
\hline $30 \mathrm{mM}$ & $5.47 \pm 0.18$ & & 12.32 \\
\hline $50 \mathrm{mM}$ & $5.87 \pm 0.44$ & & 20.53 \\
\hline $70 \mathrm{mM}$ & $6.93 \pm 0.07^{*}$ & & 42.30 \\
\hline $100 \mathrm{mM}$ & $7.00 \pm 0.04^{*}$ & & 43.74 \\
\hline $200 \mathrm{mM}$ & $7.07 \pm 0.04^{*}$ & & 45.17 \\
\hline \multicolumn{4}{|c|}{ Kunhutty } \\
\hline Control & $4.13 \pm 0.09$ & \multirow{7}{*}{0.41} & - \\
\hline $10 \mathrm{mM}$ & $4.33 \pm 0.03$ & & 4.84 \\
\hline $30 \mathrm{mM}$ & $4.67 \pm 0.07^{*}$ & & 13.08 \\
\hline $50 \mathrm{mM}$ & $5.20 \pm 0.04 *$ & & 25.91 \\
\hline $70 \mathrm{mM}$ & $5.60 \pm 0.04 *$ & & 35.59 \\
\hline $100 \mathrm{mM}$ & $5.67 \pm 0.03 *$ & & 37.29 \\
\hline $200 \mathrm{mM}$ & $5.67 \pm 0.03^{*}$ & & 37.29 \\
\hline \multicolumn{4}{|c|}{ Veliyan } \\
\hline Control & $4.20 \pm 0.13$ & \multirow{7}{*}{0.73} & - \\
\hline $10 \mathrm{mM}$ & $4.60 \pm 0.08$ & & 9.52 \\
\hline $30 \mathrm{mM}$ & $4.80 \pm 0.09$ & & 14.29 \\
\hline $50 \mathrm{mM}$ & $5.67 \pm 0.11 *$ & & 35.00 \\
\hline $70 \mathrm{mM}$ & $6.07 \pm 0.09 *$ & & 44.52 \\
\hline $100 \mathrm{mM}$ & $6.13 \pm 0.07 *$ & & 45.95 \\
\hline $200 \mathrm{mM}$ & $6.20 \pm 0.04 *$ & & 47.62 \\
\hline
\end{tabular}

*Significant variation from the control at $5 \%$ level 


\subsection{Activity of Glutathione Peroxidase}

The activity of glutathione peroxidase was found to be increasing in all the genotypes with increase in salinity level, but the change was not significant except in the case of Orkazhama (Table 5; Figs. $7 \&$ 8). It showed significant increase in enzyme activity in the case of $100 \mathrm{mM}$ and $200 \mathrm{mM}$ of $\mathrm{NaCl}$ treatments. The highest percentage of increase was noted in Kunhutty (75.47\%) followed by Orkazhama (75.00\%).

Table 5. Activity and percentage of increase in activity in the case of glutathione peroxidase in different rice cultivars under salinity stress.

\begin{tabular}{|c|c|c|c|}
\hline Cultivar/ Treatment & Mean \pm SE & CD@5\% & Percentage of increase over control \\
\hline \multicolumn{4}{|c|}{ Orthadian } \\
\hline Control & $1.27 \pm 0.05$ & \multirow{7}{*}{ NS } & - \\
\hline $10 \mathrm{mM}$ & $1.33 \pm 0.03$ & & 4.72 \\
\hline $30 \mathrm{mM}$ & $1.33 \pm 0.09$ & & 4.72 \\
\hline $50 \mathrm{mM}$ & $1.47 \pm 0.03$ & & 15.75 \\
\hline $70 \mathrm{mM}$ & $1.47 \pm 0.03$ & & 15.75 \\
\hline $100 \mathrm{mM}$ & $1.60 \pm 0.08$ & & 25.98 \\
\hline $200 \mathrm{mM}$ & $1.73 \pm 0.05$ & & 36.22 \\
\hline \multicolumn{4}{|c|}{ Chovvarian } \\
\hline Control & $1.13 \pm 0.07$ & \multirow{7}{*}{ NS } & - \\
\hline $10 \mathrm{mM}$ & $1.13 \pm 0.03$ & & - \\
\hline $30 \mathrm{mM}$ & $1.20 \pm 0.08$ & & 6.19 \\
\hline $50 \mathrm{mM}$ & $1.27 \pm 0.03$ & & 12.39 \\
\hline $70 \mathrm{mM}$ & $1.27 \pm 0.03$ & & 12.39 \\
\hline $100 \mathrm{mM}$ & $1.53 \pm 0.13$ & & 35.40 \\
\hline $200 \mathrm{mM}$ & $1.67 \pm 0.09$ & & 47.79 \\
\hline \multicolumn{4}{|c|}{ Kuttusan } \\
\hline Control & $1.20 \pm 0.04$ & \multirow{7}{*}{ NS } & - \\
\hline $10 \mathrm{mM}$ & $1.20 \pm 0.04$ & & - \\
\hline $30 \mathrm{mM}$ & $1.27 \pm 0.07$ & & 5.83 \\
\hline $50 \mathrm{mM}$ & $1.27 \pm 0.03$ & & 5.83 \\
\hline $70 \mathrm{mM}$ & $1.27 \pm 0.03$ & & 5.83 \\
\hline $100 \mathrm{mM}$ & $1.40 \pm 0.00$ & & 16.67 \\
\hline $200 \mathrm{mM}$ & $1.47 \pm 0.03$ & & 22.5 \\
\hline \multicolumn{4}{|c|}{ Kuthiru } \\
\hline Control & $1.33 \pm 0.07$ & \multirow{7}{*}{ NS } & - \\
\hline $10 \mathrm{mM}$ & $1.47 \pm 0.03$ & & 10.53 \\
\hline $30 \mathrm{mM}$ & $1.47 \pm 0.07$ & & 10.53 \\
\hline $50 \mathrm{mM}$ & $1.53 \pm 0.05$ & & 15.04 \\
\hline $70 \mathrm{mM}$ & $1.53 \pm 0.05$ & & 14.04 \\
\hline $100 \mathrm{mM}$ & $1.67 \pm 0.07$ & & 25.56 \\
\hline $200 \mathrm{mM}$ & $1.80 \pm 0.04$ & & 35.34 \\
\hline \multicolumn{4}{|c|}{ Orkazhama } \\
\hline Control & $0.80 \pm 0.04$ & \multirow{7}{*}{0.46} & - \\
\hline $10 \mathrm{mM}$ & $1.07 \pm 0.03$ & & 33.75 \\
\hline $30 \mathrm{mM}$ & $1.07 \pm 0.03$ & & 33.75 \\
\hline $50 \mathrm{mM}$ & $1.20 \pm 0.04$ & & 50.00 \\
\hline $70 \mathrm{mM}$ & $1.20 \pm 0.04$ & & 50.00 \\
\hline $100 \mathrm{mM}$ & $1.33 \pm 0.05^{*}$ & & 66.25 \\
\hline $200 \mathrm{mM}$ & $1.40 \pm 0.04 *$ & & 75.00 \\
\hline \multicolumn{4}{|c|}{ Kunhutty } \\
\hline Control & $0.53 \pm 0.03$ & \multirow{7}{*}{ NS } & - \\
\hline $10 \mathrm{mM}$ & $0.67 \pm 0.03$ & & 26.42 \\
\hline $30 \mathrm{mM}$ & $0.67 \pm 0.03$ & & 26.42 \\
\hline $50 \mathrm{mM}$ & $0.73 \pm 0.05$ & & 37.74 \\
\hline $70 \mathrm{mM}$ & $0.73 \pm 0.05$ & & 37.74 \\
\hline $100 \mathrm{mM}$ & $0.80 \pm 0.00$ & & 50.94 \\
\hline $200 \mathrm{mM}$ & $0.93 \pm 0.03$ & & 75.47 \\
\hline \multicolumn{4}{|c|}{ Veliyan } \\
\hline Control & $0.67 \pm 0.03$ & \multirow{7}{*}{ NS } & - \\
\hline $10 \mathrm{mM}$ & $0.73 \pm 0.03$ & & 8.96 \\
\hline $30 \mathrm{mM}$ & $0.80 \pm 0.04$ & & 19.40 \\
\hline $50 \mathrm{mM}$ & $0.87 \pm 0.07$ & & 29.85 \\
\hline $70 \mathrm{mM}$ & $0.87 \pm 0.07$ & & 29.85 \\
\hline $100 \mathrm{mM}$ & $1.00 \pm 0.08$ & & 49.25 \\
\hline $200 \mathrm{mM}$ & $1.13 \pm 0.05$ & & 68.66 \\
\hline
\end{tabular}

*Significant variation from the control at $5 \%$ level 


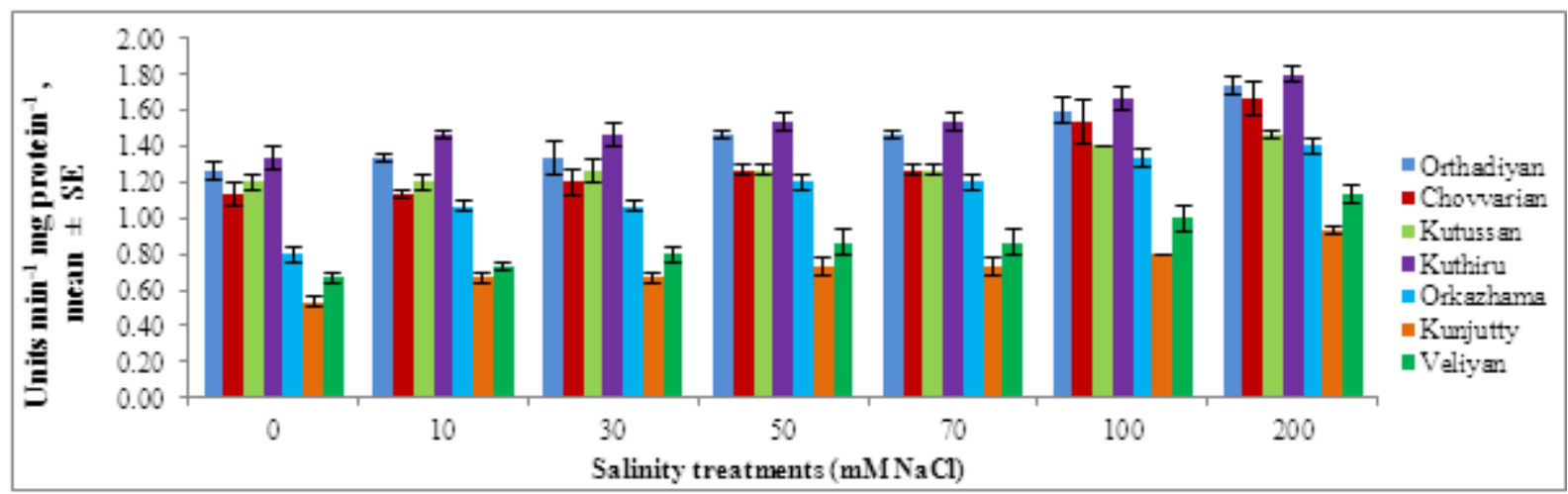

Figure 7. Activity of glutathione peroxidase in different rice genotypes under different salt concentrations

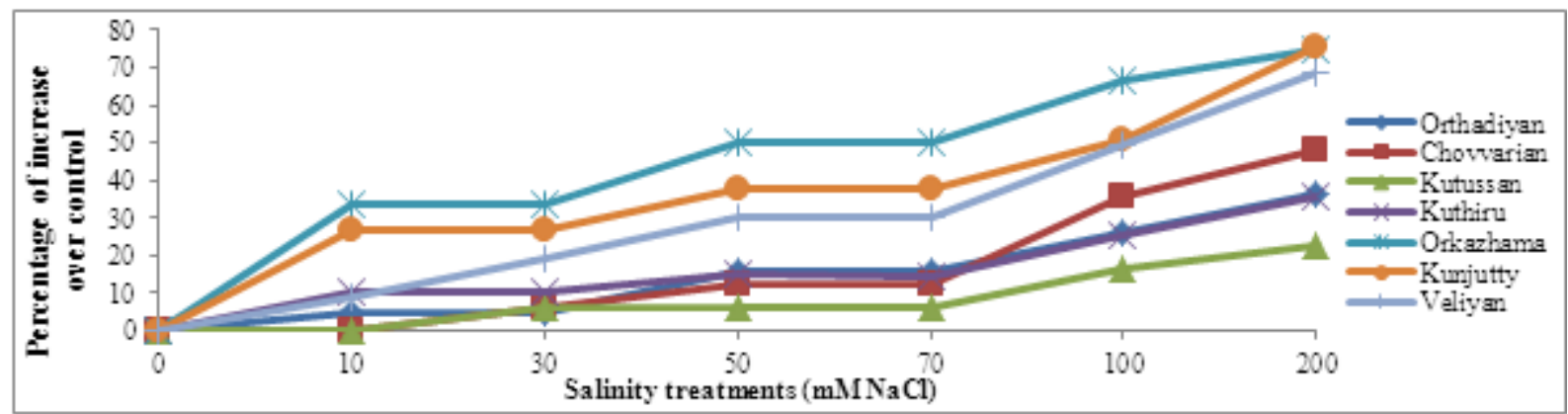

Figure 8. Increase in glutathione peroxidase activity in different rice genotypes under different salt concentrations

\subsection{Activity of ascorbate peroxidase}

Ascorbate peroxidase activity was also found to increase in relation to the increase of salinity stress (Table 6; Figs. $9 \& 10$ ). All the cultivars showed significant increase in enzyme activity starting from $30 \mathrm{mM}$ concentration of salinity stress. The highest percentage of increase was noted in Orkazhama (63.57\%) followed by Veliyan (60.88\%).

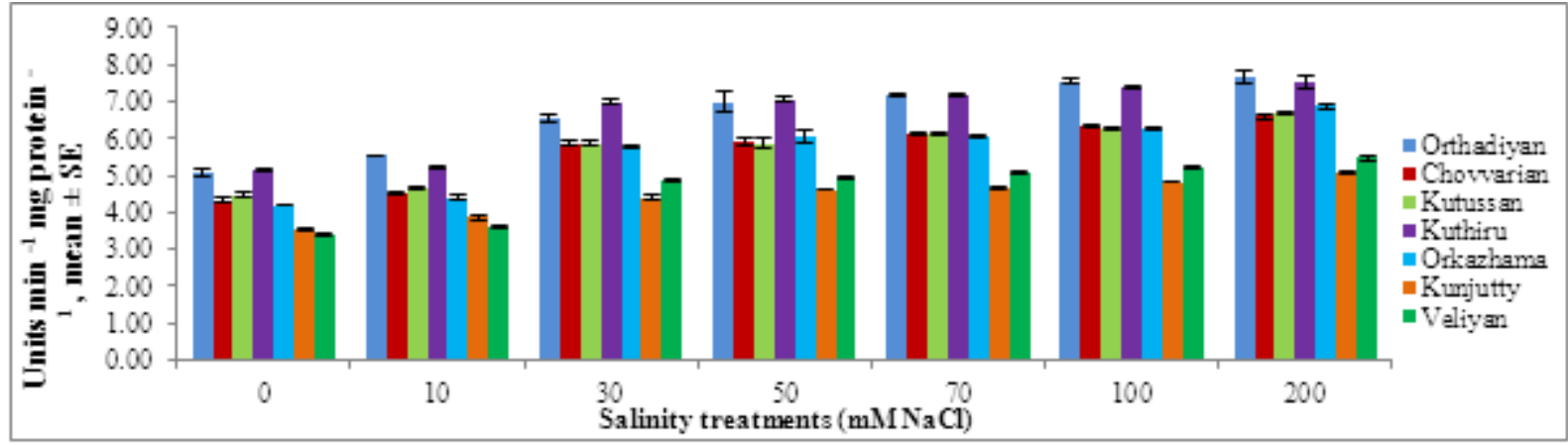

Figure 9. Activity of ascorbate peroxidase in different rice genotypes under different salt concentrations

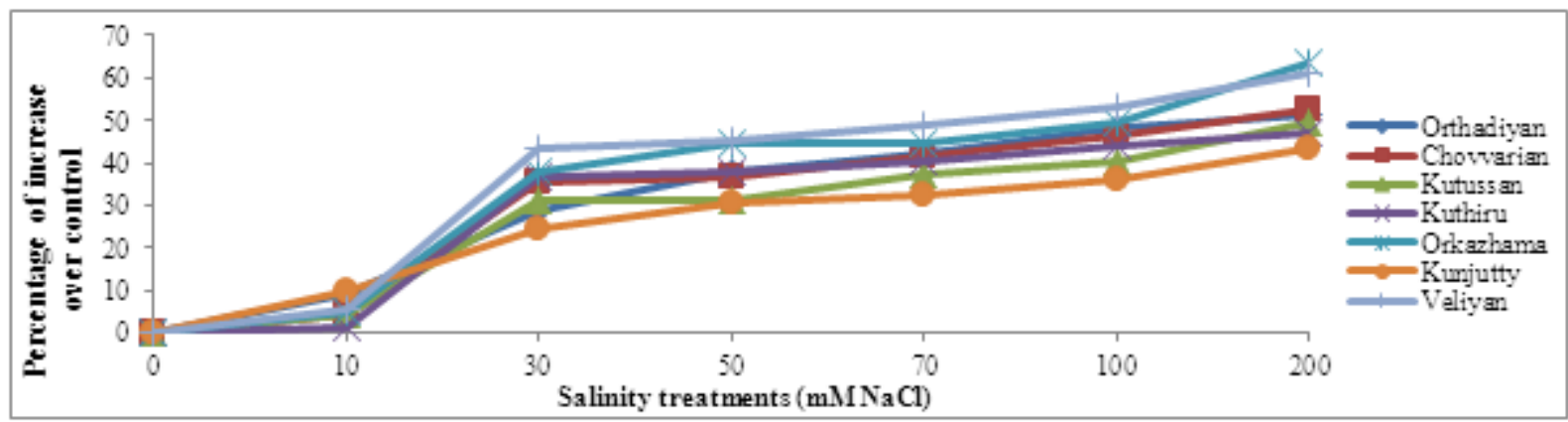

Figure 10. Increase in ascorbate peroxidase activity in different rice genotypes under different salt concentrations 
Table 6. Activity and percentage of increase in activity in the case of ascorbate peroxidase peroxidase in different rice cultivars under salinity stress.

\begin{tabular}{|c|c|c|c|}
\hline Cultivar/ Treatment & Mean \pm SE & $\mathrm{CD} @ 5 \%$ & Percentage of increase over control \\
\hline \multicolumn{4}{|c|}{ Orthadian } \\
\hline Control & $5.07 \pm 0.09$ & \multirow{7}{*}{1.07} & - \\
\hline $10 \mathrm{mM}$ & $5.53 \pm 0.03$ & & 9.07 \\
\hline $30 \mathrm{mM}$ & $6.53 \pm 0.09 *$ & & 28.80 \\
\hline $50 \mathrm{mM}$ & $7.00 \pm 0.27 *$ & & 38.07 \\
\hline $70 \mathrm{mM}$ & $7.20 \pm 0.04^{*}$ & & 42.01 \\
\hline $100 \mathrm{mM}$ & $7.53 \pm 0.07 *$ & & 48.52 \\
\hline $200 \mathrm{mM}$ & $7.67 \pm 0.18^{*}$ & & 51.28 \\
\hline \multicolumn{4}{|c|}{ Chovvarian } \\
\hline Control & $4.33 \pm 0.07$ & \multirow{7}{*}{0.53} & - \\
\hline $10 \mathrm{mM}$ & $4.53 \pm 0.03$ & & 4.62 \\
\hline $30 \mathrm{mM}$ & $5.87 \pm 0.09 *$ & & 35.57 \\
\hline $50 \mathrm{mM}$ & $5.93 \pm 0.09 *$ & & 36.95 \\
\hline $70 \mathrm{mM}$ & $6.13 \pm 0.03 *$ & & 41.57 \\
\hline $100 \mathrm{mM}$ & $6.33 \pm 0.05^{*}$ & & 46.19 \\
\hline $200 \mathrm{mM}$ & $6.60 \pm 0.08^{*}$ & & 52.42 \\
\hline \multicolumn{4}{|c|}{ Kuttusan } \\
\hline Control & $4.47 \pm 0.05$ & \multirow{7}{*}{0.55} & - \\
\hline $10 \mathrm{mM}$ & $4.67 \pm 0.03$ & & 4.47 \\
\hline $30 \mathrm{mM}$ & $5.87 \pm 0.05^{*}$ & & 31.32 \\
\hline $50 \mathrm{mM}$ & $5.87 \pm 0.14^{*}$ & & 31.32 \\
\hline $70 \mathrm{mM}$ & $6.13 \pm 0.05^{*}$ & & 37.14 \\
\hline $100 \mathrm{mM}$ & $6.27 \pm 0.07^{*}$ & & 40.27 \\
\hline $200 \mathrm{mM}$ & $6.67 \pm 0.03^{*}$ & & 49.22 \\
\hline \multicolumn{4}{|c|}{ Kuthiru } \\
\hline Control & $5.13 \pm 0.03$ & \multirow{7}{*}{0.56} & - \\
\hline $10 \mathrm{mM}$ & $5.20 \pm 0.04$ & & 1.36 \\
\hline $30 \mathrm{mM}$ & $7.00 \pm 0.04 *$ & & 36.45 \\
\hline $50 \mathrm{mM}$ & $7.07 \pm 0.05^{*}$ & & 37.82 \\
\hline $70 \mathrm{mM}$ & $7.20 \pm 0.04^{*}$ & & 40.35 \\
\hline $100 \mathrm{mM}$ & $7.40 \pm 0.04 *$ & & 44.25 \\
\hline $200 \mathrm{mM}$ & $7.53 \pm 0.14 *$ & & 46.78 \\
\hline \multicolumn{4}{|c|}{ Orkazhama } \\
\hline Control & $4.20 \pm 0.00$ & \multirow{7}{*}{0.64} & - \\
\hline $10 \mathrm{mM}$ & $4.40 \pm 0.08$ & & 4.76 \\
\hline $30 \mathrm{mM}$ & $5.80 \pm 0.04 *$ & & 38.10 \\
\hline $50 \mathrm{mM}$ & $6.07 \pm 0.18^{*}$ & & 44.52 \\
\hline $70 \mathrm{mM}$ & $6.07 \pm 0.03^{*}$ & & 44.52 \\
\hline $100 \mathrm{mM}$ & $6.27 \pm 0.05^{*}$ & & 49.30 \\
\hline $200 \mathrm{mM}$ & $6.87 \pm 0.05^{*}$ & & 63.57 \\
\hline \multicolumn{4}{|c|}{ Kunhutty } \\
\hline Control & $3.53 \pm 0.07$ & \multirow{7}{*}{0.38} & - \\
\hline $10 \mathrm{mM}$ & $3.87 \pm 0.07$ & & 9.63 \\
\hline $30 \mathrm{mM}$ & $4.40 \pm 0.08^{*}$ & & 24.65 \\
\hline $50 \mathrm{mM}$ & $4.60 \pm 0.00 *$ & & 30.31 \\
\hline $70 \mathrm{mM}$ & $4.67 \pm 0.03 *$ & & 32.29 \\
\hline $100 \mathrm{mM}$ & $4.80 \pm 0.00 *$ & & 35.98 \\
\hline $200 \mathrm{mM}$ & $5.07 \pm 0.03^{*}$ & & 43.63 \\
\hline \multicolumn{4}{|c|}{ Veliyan } \\
\hline Control & $3.40 \pm 0.04$ & \multirow{7}{*}{0.38} & - \\
\hline $10 \mathrm{mM}$ & $3.60 \pm 0.04$ & & 5.88 \\
\hline $30 \mathrm{mM}$ & $4.87 \pm 0.05^{*}$ & & 43.24 \\
\hline $50 \mathrm{mM}$ & $4.93 \pm 0.07 *$ & & 45.00 \\
\hline $70 \mathrm{mM}$ & $5.07 \pm 0.03^{*}$ & & 49.12 \\
\hline $100 \mathrm{mM}$ & $5.20 \pm 0.04 *$ & & 52.94 \\
\hline $200 \mathrm{mM}$ & $5.47 \pm 0.05^{*}$ & & 60.88 \\
\hline
\end{tabular}

*Significant variation from the control at $5 \%$ level 


\subsection{Activity of Superoxide Dismutase}

The activity of superoxide dismutase also increased significantly in all the rice cultivars studied in relation to salt stress (Table 7; Figs. 11 \& 12). Except Orthadian, all the other cultivars showed significant increase in superoxide dismutase activity from $30 \mathrm{mM}$ salt concentration. The cultivar Kunhutty showed the highest percentage of increase in SOD activity (206.03\%) followed by Veliyan (258.39\%) and both of these are cultivars from the non saline habitat.

Table 7. Activity and percentage of increase in activity in the case of superoxide dismutase in different rice cultivars under salinity stress.

\begin{tabular}{|c|c|c|c|}
\hline Cultivar/ Treatment & Mean \pm SE & CD@M5\% & $\begin{array}{l}\text { Percentage of increase over } \\
\text { control }\end{array}$ \\
\hline \multicolumn{4}{|c|}{ Orthadian } \\
\hline Control & $15.80 \pm 0.39$ & \multirow{7}{*}{1.95} & - \\
\hline $10 \mathrm{mM}$ & $16.87 \pm 0.35$ & & 6.77 \\
\hline $30 \mathrm{mM}$ & $17.33 \pm 0.14$ & & 9.68 \\
\hline $50 \mathrm{mM}$ & $19.93 \pm 0.14^{*}$ & & 26.14 \\
\hline $70 \mathrm{mM}$ & $22.93 \pm 0.03 *$ & & 45.13 \\
\hline $100 \mathrm{mM}$ & $24.20 \pm 0.19^{*}$ & & 53.16 \\
\hline $200 \mathrm{mM}$ & $25.33 \pm 0.25^{*}$ & & 60.32 \\
\hline \multicolumn{4}{|c|}{ Chovvarian } \\
\hline Control & $12.67 \pm 0.47$ & \multirow{7}{*}{2.36} & - \\
\hline $10 \mathrm{mM}$ & $13.80 \pm 0.35$ & & 8.92 \\
\hline $30 \mathrm{mM}$ & $16.00 \pm 0.23^{*}$ & & 26.28 \\
\hline $50 \mathrm{mM}$ & $18.53 \pm 0.15^{*}$ & & 46.25 \\
\hline $70 \mathrm{mM}$ & $22.53 \pm 0.30^{*}$ & & 77.82 \\
\hline $100 \mathrm{mM}$ & $23.13 \pm 0.28^{*}$ & & 82.56 \\
\hline $200 \mathrm{mM}$ & $26.80 \pm 0.13^{*}$ & & 111.52 \\
\hline \multicolumn{4}{|c|}{ Kuttusan } \\
\hline Control & $11.13 \pm 0.24$ & \multirow{7}{*}{2.02} & - \\
\hline $10 \mathrm{mM}$ & $11.87 \pm 0.29$ & & 6.65 \\
\hline $30 \mathrm{mM}$ & $14.20 \pm 0.20^{*}$ & & 27.58 \\
\hline $50 \mathrm{mM}$ & $17.13 \pm 0.10^{*}$ & & 53.91 \\
\hline $70 \mathrm{mM}$ & $21.13 \pm 0.22 *$ & & 89.85 \\
\hline $100 \mathrm{mM}$ & $22.27 \pm 0.22 *$ & & 100.09 \\
\hline $200 \mathrm{mM}$ & $24.40 \pm 0.39 *$ & & 119.23 \\
\hline \multicolumn{4}{|c|}{ Kuthiru } \\
\hline Control & $17.47 \pm 0.17$ & \multirow{7}{*}{1.88} & - \\
\hline $10 \mathrm{mM}$ & $18.20 \pm 0.23$ & & 4.18 \\
\hline $30 \mathrm{mM}$ & $20.40 \pm 0.33 *$ & & 16.77 \\
\hline $50 \mathrm{mM}$ & $22.60 \pm 0.15^{*}$ & & 29.36 \\
\hline $70 \mathrm{mM}$ & $25.87 \pm 0.05^{*}$ & & 48.08 \\
\hline $100 \mathrm{mM}$ & $26.20 \pm 0.27 *$ & & 49.97 \\
\hline $200 \mathrm{mM}$ & $28.40 \pm 0.31 *$ & & 62.56 \\
\hline \multicolumn{4}{|c|}{ Orkazhama } \\
\hline Control & $11.33 \pm 0.21$ & \multirow{7}{*}{1.42} & - \\
\hline $10 \mathrm{mM}$ & $11.60 \pm 0.15$ & & 2.38 \\
\hline $30 \mathrm{mM}$ & $13.47 \pm 0.24 *$ & & 18.89 \\
\hline $50 \mathrm{mM}$ & $15.53 \pm 0.22 *$ & & 30.07 \\
\hline $70 \mathrm{mM}$ & $19.13 \pm 0.18^{*}$ & & 68.84 \\
\hline $100 \mathrm{mM}$ & $23.07 \pm 0.18^{*}$ & & 103.62 \\
\hline $200 \mathrm{mM}$ & $25.00 \pm 0.04 *$ & & 120.65 \\
\hline \multicolumn{4}{|c|}{ Kunhutty } \\
\hline Control & $7.80 \pm 0.19$ & \multirow{7}{*}{1.21} & - \\
\hline $10 \mathrm{mM}$ & $8.53 \pm 0.22$ & & 9.36 \\
\hline $30 \mathrm{mM}$ & $9.33 \pm 0.13 *$ & & 19.62 \\
\hline $50 \mathrm{mM}$ & $13.33 \pm 0.11 *$ & & 70.90 \\
\hline $70 \mathrm{mM}$ & $18.00 \pm 0.08^{*}$ & & 130.77 \\
\hline $100 \mathrm{mM}$ & $20.60 \pm 0.19^{*}$ & & 164.10 \\
\hline $200 \mathrm{mM}$ & $23.87 \pm 0.07 *$ & & 206.03 \\
\hline \multicolumn{4}{|c|}{ Veliyan } \\
\hline Control & $9.47 \pm 0.05$ & \multirow{7}{*}{1.69} & - \\
\hline $10 \mathrm{mM}$ & $10.93 \pm 0.13$ & & 15.42 \\
\hline $30 \mathrm{mM}$ & $12.20 \pm 0.04 *$ & & 28.83 \\
\hline $50 \mathrm{mM}$ & $14.80 \pm 0.12 *$ & & 56.28 \\
\hline $70 \mathrm{mM}$ & $19.07 \pm 0.20^{*}$ & & 101.37 \\
\hline $100 \mathrm{mM}$ & $21.20 \pm 0.37 *$ & & 123.86 \\
\hline $200 \mathrm{mM}$ & $24.47 \pm 0.31^{*}$ & & 158.39 \\
\hline
\end{tabular}

*Significant variation from the control at $5 \%$ level 


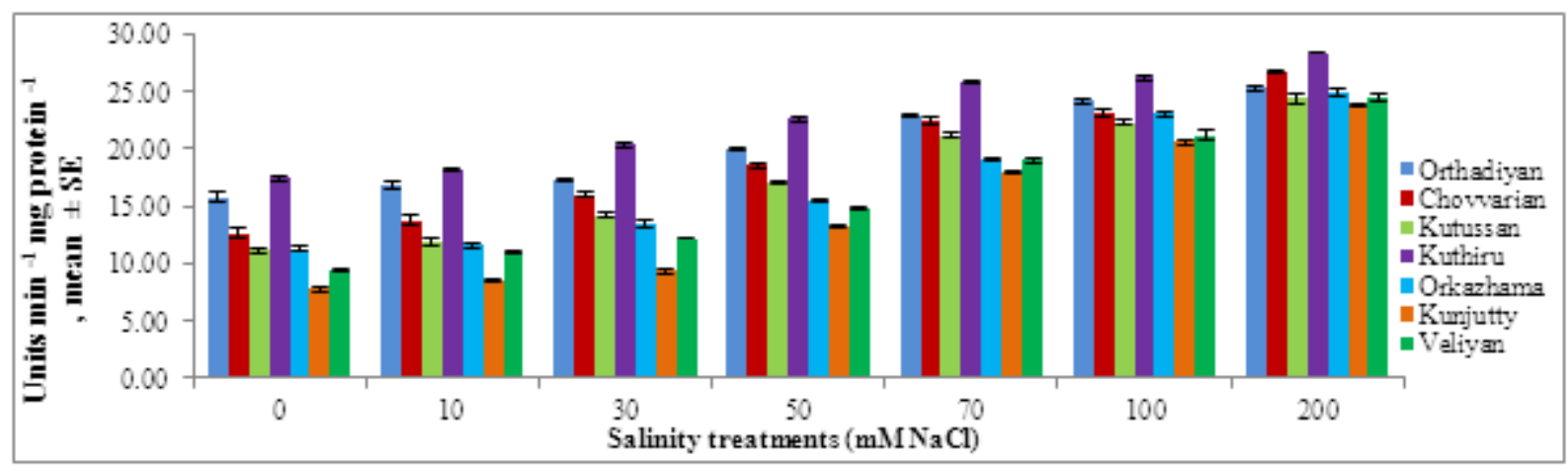

Figure 11. Activity of superoxide dismutase in different rice genotypes under different salt concentrations

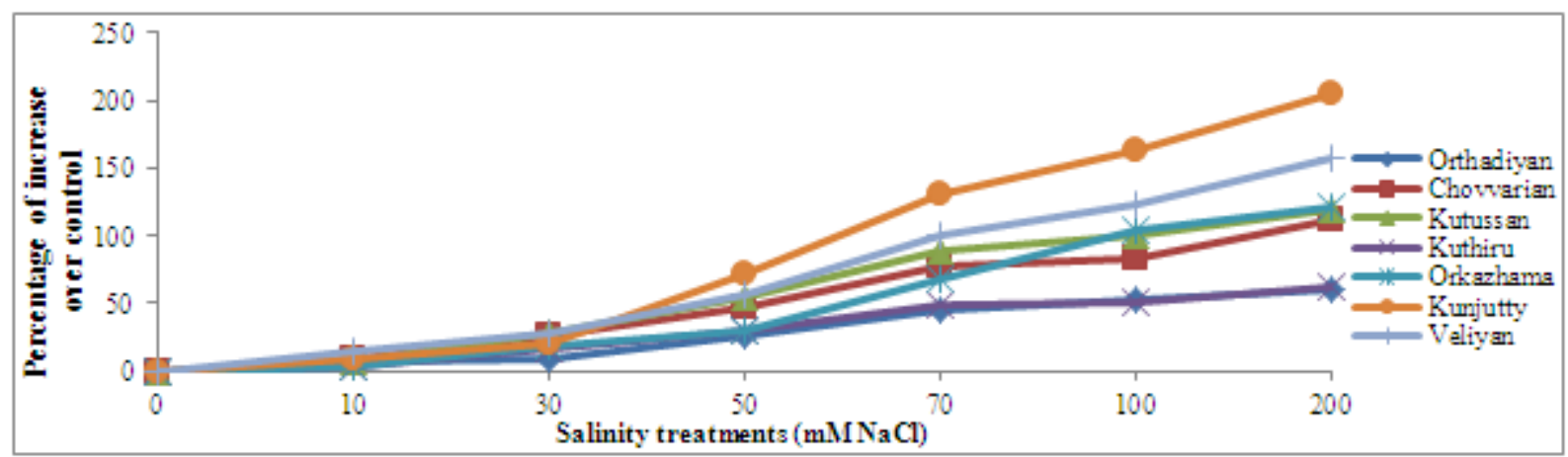

Figure 12. Increase in superoxide dismutase activity in different rice genotypes under different salt concentrations

\section{Discussion}

It is suggested that in salt tolerant plants, increased activity of anti-oxidative enzymes is responsible for reducing $\mathrm{H}_{2} \mathrm{O}_{2}$ levels. Salinity tolerant varieties will be one of the remedy to withstand increasing soil salinity globally. Such efficient varieties should have effective antioxidant mechanisms to adapt to the adverse conditions. So detailed knowledge on the physiological, genetic and molecular mechanisms of salt resistance will be one of the strongest tools for crop/rice breeding programs with this perspective [51]. The enhanced quantities of different ROS can be viewed as a threat to cells because it produces higher ROS such as hydroxyl radical $\left(\mathrm{HO}^{-}\right)$which attack proteins, lipids and nucleic acids and act as secondary messengers involved in the stress response signal transduction pathway [39]. $\mathrm{H}_{2} \mathrm{O}_{2}$ is produced during different metabolic processes such as photorespiration in mitochondria and chloroplasts [52].

Catalase and peroxidase are the $\mathrm{H}_{2} \mathrm{O}_{2}$ detoxifying enzymes and mostly associated with peroxisomes where they remove $\mathrm{H}_{2} \mathrm{O}_{2}$ formed during photorespiration. Peroxidases are another group of non-chloroplastic enzymes that detoxify $\mathrm{H}_{2} \mathrm{O}_{2}$ in the cell cytosol. All the cultivars used in our study also showed significantly increased level of peroxidase and catalase activity under salt stress. Earlier workers [53] have also reported an increase in peroxidase activity in rice cultivars under salt stress. Our data also reveal that among all the cultivars studied, the candidates from the traditional saline rice tracts showed the highest quantum of catalase and peroxidase activity due to increase in salinity stress.
Glutathione reductase (GR) exhibited a cumulative trend in all the cultivars under increasing salt stress. GR is a flavo-protein containing oxido reductase found in both prokaryotes and eukaryotes [54]. It is a potential enzyme of the ASH-GSH cycle and plays an essential role in defence system against Reactive Oxygen Species by sustaining the reduced status of GSH and confers abiotic stress tolerance in plants [55]. It is done efficiently by maintaining the reduced GSH pool through catalyzing the reduction of GSSG to GSH with the accompanying oxidation of NADPH in the cell [56]. It is localized predominantly in chloroplasts, but small amount of this enzyme has been found in mitochondria and cytosol also. More than $80 \%$ of its activity in photosynthetic tissues was reported to be of chloroplastic [57,58]. Among the rice cultivars studied presently, GR activity is higher in Kuthiru, a genotype collected ted from a traditional saline rice tract. Our results reveal a diminished activity of GR in rice cultivars collected from non sline rice tract (Kunhutty and Veliyan) when compared to others. Uddin et al. (2007) [59] identified mutant lines of rice that showed high tolerance to salinity and higher induction of APX, GR, and SOD. These rice mutant lines indicated that they have the ability to up-regulate their anti-oxidative system in order to cope up with salt-induced stress. In the present study, the cultivars Kuthiru and Orthadian showed highest activity of glutathione reductase activity and they are the representatives of the traditional rice cultivars of the saline rice tracts of North Kerala. It has already been reported that in terms of growth and yiled parameters, they perform well under low to medium salinity levels [60]. 
Glutathione peroxidases (GPX) are a large family of diverse isozymes that use GSH to reduce $\mathrm{H}_{2} \mathrm{O}_{2}$ and organic and lipid hydroperoxides, and therefore help plant cells from oxidative stress [61]. Glutathione peroxidase uses reduced glutathione as reductant, thus providing an alternate line of defense in addition to the reaction catalyzed by ascorbate peroxidase (APX). Higher activity of GPX was noted in Kuthiru, a cultivar collected from the saline rice tract as compared to other genotypes. It is suggested that this may also be one of the reasons for the efficient antioxidant defense system in salt tolerant rice varieties and may be one of the contributing factors conferring salt tolerance. Our data shows that GPX is significantly increased only in Orkazhama under salinity stress; a genotype from the traditional saline system.

Ascorbate peroxidase (APX) is another important antioxidant enzyme in plants that detoxify hydrogen peroxide using ascorbate as reductant. APX exists in several isoforms found in various compartments of plant cells and shows differential expression and regulation under environmental stress factors [62]. APX is involved in the scavenging mechanism of $\mathrm{H}_{2} \mathrm{O}_{2}$ formed in water-water and ASH-GSH cycles and utilizes ASH as the electron donor. The APX family consists of at least five different isoforms including thylakoid (tAPX) and glyoxisome membrane forms (gmAPX), chloroplast stromal soluble form (sAPX) and cytosolic form (cAPX) [63]. APX has a higher affinity for $\mathrm{H}_{2} \mathrm{O}_{2}$ than CAT and POX and it may have a more crucial role in the management of ROS during stress. Enhanced expression of APX in plants has been demonstrated during different stress conditions. Hsu and Kao (2007) [64] reported that pre-treatment of Oryza sativa seedlings with $\mathrm{H}_{2} \mathrm{O}_{2}$ under non-heat shock conditions resulted in an increase in APX activity and protected rice seedlings from subsequent $\mathrm{Cd}$ stress. Sharma and Dubey (2005) [65] found that mild drought stressed plants had higher chloroplastic APX activity than control grown plants but the activity declined at higher levels of drought stress. It has been suggested that a decrease in enzyme activity may be due to an imbalance between production of ROS and the quenching ability of antioxidants. During the present study, the activity of APX increased significantly in $\mathrm{NaCl}$ exposed rice plants, collected from both saline and non-saline rice tracts.

SOD is the most effective intracellular enzymatic antioxidant which is ubiquitous in all aerobic organisms and in all subcellular compartments prone to ROS mediated oxidative stress. It is well established that various environmental stresses often lead to the increased generation of ROS, where, SOD has been proposed to be important in plant stress tolerance and it provides the first line of defense against the toxic effects of elevated levels of ROS. The up regulation of SODs is implicated in combating oxidative stress caused due to biotic and abiotic stress and have a critical role in the survival of plants under stresses environment. Earlier studies have shown that salt-tolerant rice plants exhibited an elevated level of SOD activity, whereas relatively low activity was found in sensitive plants
[66,67]. In the present work, the highest activity of SOD was noticed in Kuthiru, a cultivar from the saline rice tract and Kunhutty shows highest percentage of increase in enzyme activity $(206.03 \%)$ from the control plants. It is suggested that in salt tolerant plants, increased activity of anti-oxidative enzymes is responsible for reducing $\mathrm{H}_{2} \mathrm{O}_{2}$ levels, inhibiting membrane damage and lowering lipid peroxidation and therefore salt tolerant plants are better protected from oxidative damage under salt stress compared to salt sensitive plants [68]. The genotypes from the traditional saline system showed the highest antioxidant enzyme activity under salinity stress. The production and release of ROS may be dramatically enhanced when plants are exposed to a wide range of environmental contaminants [69]. Mittal and Dubey (1991) [53] suggested that salinity affects mainly the de novo synthesis of the enzyme since inhibition under in vitro conditions and activation under in vivo conditions have been observed in salt sensitive cultivars. The increase in the activity of these enzymes may be one of the reasons for the survival nature of the varieties under salinity stress, since the salt tolerant rice varieties show highest antioxidant activity.

\section{Conclusions}

With the ultimate goal to develop rice varieties with enhanced suitability towards the changing environmental conditions, intensive efforts are going on worldwide using physiological, biochemical and molecular tools. In conclusion, results of the present study indicate that rice cultivars differing in salt tolerance show varying levels of ROS scavenging anti-oxidative enzyme production and activity levels when grown under salinity. The salt tolerance property in rice seedlings is associated with higher levels of the various antioxidants and higher activity levels of the anti-oxidative enzymes SOD, CAT, POX GPX, APX, and GR. These observations further suggest that the status of antioxidants and anti-oxidative enzymes in the protoplasm can serve as major determinants for developing salt tolerance in rice.

\section{REFERENCES}

[1] Duncan, R.R. and R.N. Carrow, 2001. Molecular breeding for tolerance to abiotic/edaphic stresses in forage and turf grass. In: Spangenberg, G. (ed.). Molecular Breeding of Forage Crops. Kluwer Academic Publishers, Dordrecht, pp: 251-260.

[2] Shah, K., R.G. Kumar, S. Verma and R.S. Dubey, 2001. Effect of cadmium on lipid peroxidation, superoxide anion generation and activities of antioxidant enzymes in growing rice seedlings. Plant Sci. 161: 1135-1144.

[3] Verma, S. and R.S. Dubey, 2003. Lead toxicity induces lipid peroxidation and alters the activities of antioxidant enzymes in growing rice plants. Plant Sci. 164: 645-655. 
[4] Kochhar, S. and V.K. Kochhar, 2005. Expression of antioxidant enzymes and heat shock proteins in relation to combined stress of cadmium and heat in Vigna mungo seedlings. Plant Sci. 168: 921-929.

[5] Epstein, E., J.D. Norlyn, D.W. Rush, R.W. Kingsbury, D.B. Kelley, G.A. Cunningham and A.F. Wrona, 1980. Saline culture of crops: a genetic approach. Science 210: 399-404.

[6] Greenway, H. and R. Munns, 1980. Mechanism of salt tolerance in non-halophytes. Annu. Rev. Plant Physiol. 31: 149-190.

[7] Yancey, P.H., M.E. Clark, S.C. Hand, R.D. Bowlus and G.M. Somero, 1982. Living with water stress: evolution of osmolyte system. Science 217: 1214-1222.

[8] Dubey, R.S., 1999. Protein synthesis by plants under stressful conditions. In: Pessarakli, M. (Ed.), Handbook of Plant and Crop Stress. Marcel Dekker, New York, USA. pp: 365-397.

[9] Hussain, K., M.F. Nisar, A. Majeed, K. Nawaz, K.H. Bhatti, S. Afghan, A. Shahazad and S.Z. Hussnian, 2010. What molecular mechanism is adapted by plants during salt stress tolerance? Afr. J. Biotechnol. 9: 416-422.

[10] Suzuki, N. and R. Mittler, 2006. Reactive oxygen species and temperature stresses: A delicate balance between signaling and destruction. Physiol. Plant. 126: 45-51.

[11] Bohnert, H.J., D.E. Nelson and R.G. Jensen, 1995. Adaptations to environmental stresses. Plant Cell 7: 1099-1111(1995).

[12] Hasegawa, P.M., R.A. Bressan, J.K. Zhu and H.J. Bohnert, 2000. Plant cellular and molecular responses to high salinity. Annu. Rev. Plant Physiol. Plant Mol. Biol. 51: 463-499.

[13] Zhu, J.K., 2002. Salt and drought stress signal transduction in plants. Annu. Rev. Plant Biol. 53: 247-273.

[14] Dubey, R.S., 1994. Protein synthesis by plants under stressful conditions. In: Pessarakli, M. (ed.). Handbook of Plant and Crop Stress. Marcel Dekker, New York, USA. pp. 277-299

[15] Richharia, A., K. Shah and R.S. Dubey, 1997. Nitrate reductase from rice seedlings: partial purification, characterization and the effects of in situ and in vitro $\mathrm{NaCl}$ salinity. J. Plant Physiol. 151(3): 316-322.

[16] Dubey, R.S., 1997. Protein synthesis by plants under stressful conditions. In: Pessarakli, M. (ed.). Handbook of Plant and Crop Stress. Marcel Dekker, New York, USA. pp. 859-875.

[17] Khan, M.H. and S.K. Panda, 2008. Alterations in root lipid peroxidation and antioxidative responses in two rice cultivars under NaCl-salinity stress. Acta Physiol. Plant. 30: 81-89.

[18] Khush, G.S., 2005. What will it take to feed 5.0 million rice consumers in 2030. Plant Mol. Biol. 59: 1-6.

[19] Akbar, M. and F.N. Ponnamperuma, 1980. Saline soil of south and southeast Asia as potential rice lands. In: Rice Research Strategies for the Future, IRRI, Manila, Philippines. pp. 265-281.

[20] Flowers, T.J. and A.R. Yeo, 1989. Effects of salinity on plant growth and crop yield. In: Cherry, J.H. (ed.).,
Environmental Stress in Plants. Springer Verlag, Berlin. pp. 101-119.

[21] Gao, J.P., D.Y. Chao and H.H. Lin, 2007. Understanding abiotic stress tolerance mechanisms: recent studies on stress response in rice. J. Integr. Plant Biol. 49(6): 742-750.

[22] Serrano, R., 1996. Salt tolerance in plants and microorganisms: toxicity targets and defense responses. Int. Rev. Cytol. 165: 1-52.

[23] Takabe, T., T. Nakamura, M. Nomura, Y. Hayashi, M. Ishitani,Y. Muramoto, A. Tanaka and T. Takabe, 1998. Glycine betaine and the genetic engineering of salinity tolerance in plants, In: Satoh, K. and N. Murata (eds.). Stress Responses of Photosynthetic Organisms. Elsevier Science, Amsterdam. pp. 115-131.

[24] Volkmar, K.M., Y. Hu and H. Steppuhn, 1998. Physiological responses of plant to salinity: a review. Can. J. Plant Sci. 78: 19-27.

[25] Allen, R.G. and A.K. Balon, 1989. Oxidative influence in development and differentiation and overview of a free radical theory of development. Free Radical Bio. Med. 6: 631-661.

[26] Hockenbery, D.M., Z.N. Oltvai, X.M. Yin, C.L. Milliman and S.J. Korsmeyer, 1993. Bcl-2 functions in an antioxidant pathway to prevent apoptosis. Cell 75: 241-251.

[27] Shibanuma, M., T. Kuroki and K. Nose, 1988. Induction of DNA replication and expression of proto-oncogenes c-myc and $\mathrm{c}-\mathrm{fos}$ in quiescent Balb/3T3 cells by xanthine/xanthine oxidase. Oncogene. 3: 17-21.

[28] Lo, Y.Y.C., J.M.S. Wong and T.F. Cruz, 1996. Reactive oxygen species mediate cytokine activation of c-Jun $\mathrm{NH}_{2}$-terminal kinases. J. Biol. Chem. 271: 15703-15707.

[29] Bowler, C., M. van Montagu and D. Inze, 1992. Superoxide dismutase and stress tolerance. Annu. Rev. Plant Physiol. Plant Mol. Biol. 43: 83-116.

[30] Willekens, H., S. Chamnongpol, M. Davey, M. Schraudner, C. Langebartels, M. Van Montagu, D. Inze and W. Van Camp, 1997. Catalase is a sink for $\mathrm{H}_{2} \mathrm{O}_{2}$ and is indispensable for stress defence in $\mathrm{C}_{3}$ plants. EMBO J. 16: 4806-4816.

[31] Mori, H., K. Higo, H. Higo, Y. Minobe, H. Matsui and S. Chiba, 1992. Nucleotide and derived amino acid sequence of a catalase cDNA isolated from rice immature seeds. Plant Mol. Biol. 18: 973-976.

[32] Ito, H., N. Hiraoka, A. Ohbayashi and Y. Ohashi, 1991. Purification and characterization of rice peroxidases. Agr. Biol. Chem. 55: 2445-2454.

[33] Lee, S.H., N. Ahsan, K.W. Lee, D.H. Kim, D.G. Lee, S.S. Kwak, S.Y. Kwon, T.H. Kim and B.H. Lee, 2007. Simultaneous overexpression of both $\mathrm{Cu} / \mathrm{Zn}$ superoxide dismutase and ascorbate peroxidase in transgenic tall fescue plants confers increased tolerance to a wide range of abiotic stresses. J. Plant Physiol. 64: 1626-1638.

[34] Foyer, C.H., H. Lopez-Delgado, J.F. Dat and I.M. Scott, 1997. Hydrogen peroxide and glutathione-associated mechanisms of acclamatory stress tolerance and signalling. Physiol. Plant. 100: 241-254.

[35] Apse, M.P. and E. Blumwald, 2002. Engineering salt tolerance in plants. Curr. Opin. Biotech. 13: 146-150. 
[36] Ahmed S., E. Nawata, M. Hosokawa, Y. Domae and T. Sakuratani, 2002. Alterations in photosynthesis and some antioxidant enzymatic activities of mung bean subjected to water logging. Plant Sci. 163: 117-123.

[37] Dewir, Y.H., D. Chakrabarty, M.B. Ali, E.J. Hahn and K.Y. Paek, 2006. Lipid peroxidation and antioxidant enzyme activities of Euphorbia millii hyperhydric shoots. Environ. Exp. Bot. 58: 93-99.

[38] Asada, K., 1992. Ascorbate peroxidase-a hydrogen peroxide scavenging enzyme in plants. Plant Physiol. 85: 235-241.

[39] Foyer, C., P. Descourvieres and K.J. Kunert, 1994. Protection against oxygen radicals: an important defense mechanism studied in transgenic plant. Plant Cell Environ. 17: $507-523$.

[40] Asada, K., 1999. The water-water cycle in chloroplasts: scavenging of active oxygen and dissipation of excess photons. Annu. Rev. Plant Physiol. Plant Mol. Biol. 50: 601-639.

[41] Larkindale, J. and B. Huang, 2004. Thermo tolerance and antioxidant systems in Agrostis stolonifera: Involvement of salicylic acid, abscisic acid, calcium, hydrogen peroxide and ethylene. J. Plant Physiol. 161: 405-413.

[42] Aebi, H., 1983. Catalase. In: Bergmeies, H. (ed.). Methods of Enzymatic Analysis. Verlag Chemie Wenhein, Adamse, Germany. pp. 273-277.

[43] Rao, M.V., G. Paliyath and D.P. Ormrod, 1996. Ultraviolet-B- and ozone induced biochemical changes in antioxidant enzymes of Arabidopsis thaliana. Plant Physiol. 110: 125-136.

[44] Foyer, C.H. and B. Halliwell, 1986. The presence of glutathione and glutathione reductase in chloroplasts: a proposed role in ascorbic acid metabolism. Planta. 133: 21-25.

[45] Smith, I.K., T.L. Vierheller and C.A. Thorne, 1988. Assay of glutathione reductase in crude tissue homogenates using 5, 5'-dithiobis (2-nitrobenzoic acid). Anal. Biochem. 175: 408-413.

[46] Flohe, L and W.A. Gunzler, 1984. Assays of glutathione peroxidase. Method Enzymol. 105: 114-121.

[47] Asada, K., 1994. Production and action of active oxygen species in photosynthetic tissues.In: Foyer, C.H. and P. Mullineaux (eds.). Causes of photo-oxidative stress and amelioration of defense systems in plants. CRC Press, Boca Raton, FL. pp. 77-104.

[48] Nakano, Y. and K. Asada, 1981. Hydrogen peroxide is scavenged by ascorbate specific peroxidase in spinach chloroplast. Plant Cell Physiol. 22: 867-880.

[49] Beyer, W.F. and I. Fridovich, 1987. Assaying for superoxide dismutase activity: some large consequences of minor changes in conditions. Anal. Biochem. 161: 559-566.

[50] Lowry, O.H., N.J. Rosebrough, A.L. Farr and R.J. Randall, 1951. Protein measurement with the Folin phenol reagent. J. Biol. Chem. 193: 265-275.

[51] Peskan-Berghöfer, T., J. Neuwirth, V. Kusnetsov and R. Oelmüller, 2005. Suppression of heterotrimeric G-protein $\beta$-subunit affects anther shape, pollen development and inflorescence architecture in tobacco. Planta. 220(5): 737-746.

[52] Henzler, T. and E. Steudle, 2000. Transport and metabolic degradation of hydrogen peroxide in Chara coralline: model calculations and measurements with the pressure probe suggest transport of $\mathrm{H}_{2} \mathrm{O}_{2}$ across water channels. J. Exp. Bot. 353: 2053-2066.

[53] Mittal, R. and R.S. Dubey, 1991. Behaviour of peroxidases in rice: changes in enzyme activity and isoforms in relation to salt tolerance. Plant Physiol. Biochem. 29: 31-40.

[54] Romero-Puertas, M.C., F.J. Corpas, L.M. Sandalio, M. Leterrier, M. Rodriguez Serrano, L.A. del Rio and J.M. Palma, 2006. Glutathione reductase from pea leaves: response to abiotic stress and characterization of the peroxisomal isozyme. New Phytol. 170: 43-52.

[55] Hasanuzzaman, M., M. Hossain, J.T. Silva and M. Fujita, 2012. Plant response and tolerance to abiotic oxidative stress: antioxidant defense is a key factor. In: Venkateswarlu, B., A.K. Shanker, C. Shanker and M. Maheswari (eds.). Crop Stress and its Management: perspectives and strategies. Springer, Netherlands. pp. 261-315.

[56] Contour-Ansel, D., M.L. Torres-Franklin, M.H.C. De Carvalho, A. D'arcy-Lameta and Y. Zuily-Fodil, 2006. Glutathione reductase in leaves of cowpea: cloning of two cDNAs, expression and enzymatic activity under progressive drought stress, desiccation and abscisic acid treatment. Ann. Bot. 98: 1279-1287.

[57] Edwards, E.A., S. Rawsthorne and P.M. Mullineaux, 1990. Subcellular distribution of multiple forms of glutathione reductase in leaves of pea (Pisum sativum L.). Planta 180: 278-284.

[58] Ashraf, M.: Biotechnological approach of improving plant salt tolerance using antioxidants as markers. Biotechnol. $A d v ., 27,84-93$ (2009).

[59] Uddin, M.I., M.H. Rashid, N. Khan, M.F. Perveen, T.H. Tai and K. Tanaka, 2007. Selection of promising salt tolerant rice mutants derived from cultivar 'drew' and their antioxidant enzymes activity under salt stress. SABRAO J. Breed. Genet. 39: 89-98.

[60] Chandramohanan, K.T. and K.V. Mohanan, 2012. Kaipad rice farming in North Kerala- an indigenous saline resistant organic farming system. Ind. J. Tradit. Knowl. 11(1): 185 -189 .

[61] Noctor, G., L. Gomez, H. Vanacker and C.H. Foyer, 2002. Interactions between biosynthesis, compartmentation and transport in the control of glutathione homeostasis and signalling. J. Exp. Bot. 53: 1283-1304.

[62] Teixeira, F.K., L. Menezes-Benavente, V.C. Galvão, R. Margis and M. Margis-Pinheiro, 2006. Rice ascorbate peroxidase gene family encodes functionally diverse isoforms localized in different subcellular compartments. Planta 224: 300-314.

[63] Noctor, G. and C.H. Foyer, 1998. Ascorbate and glutathione: Keeping active oxygen under control. Annu. Rev. Plant Physiol. Plant Mol. Biol. 49: 249-279.

[64] Hsu, Y.T. and C.H. Kao, 2007. Heat shock-mediated $\mathrm{H}_{2} \mathrm{O}_{2}$ accumulation and protection against $\mathrm{Cd}$ toxicity in rice seedlings. Plant Soil. 300: 137-147. 
[65] Sharma, P. and R.S. Dubey, 2005. Modulation of nitrate reductase activity in rice seedlings under aluminium toxicity and water stress: role of osmolytes as enzyme protectant. J. Plant Physiol. 162: 854-864 (2005).

[66] Dionisio-Sese, M.L. and S. Tobita, 1998. Antioxidant responses of rice seedlings to salinity stress. Plant Sci. 135: $1-9$.

[67] Bhattacharjee, S. and A.K. Mukherjee, 1997. Role of free radicals in membrane deterioration in three rice (Oryza sativa L.) cultivars under $\mathrm{NaCl}$ salinity at early germination stage. Ind. J. Exp. Bot. 35: 1365-1369.

[68] Azevedo-Neto, A.D., J.T. Prisco, J. Eneas-Filho, C.E.B. de Abrau and E. Gomez-Filho, 2006. Effect of salt stress on anti-oxidative enzymes and lipid peroxidation in leaves and roots of salt-tolerant and salt sensitive maize genotypes. Environ. Exp. Bot. 56: 87-94.

[69] Mittler, R., 2002. Oxidative stress, antioxidants and stress tolerance. Trends Plant Sci. 7: 405-410. 